\title{
Finite-time Lyapunov dimension and hidden attractor of the Rabinovich system
}

\author{
N. V. Kuznetsov • G. A. Leonov • T. N. Mokaev • \\ A. Prasad • M. D. Shrimali
}

Received: 18 November 2017 / Accepted: 1 January 2018 / Published online: 31 January 2018

(C) The Author(s) 2018. This article is an open access publication

\begin{abstract}
The Rabinovich system, describing the process of interaction between waves in plasma, is considered. It is shown that the Rabinovich system can exhibit a hidden attractor in the case of multistability as well as a classical self-excited attractor. The hidden attractor in this system can be localized by analytical/numerical methods based on the continuation and perpetual points. The concept of finite-time Lyapunov dimension is developed for numerical study of the dimension of attractors. A conjecture on the Lyapunov dimension of self-excited attractors and the notion of exact Lyapunov dimension are discussed. A comparative survey on the computation of the finite-time Lyapunov exponents and dimension by different algorithms is presented. An adaptive algorithm for studying the dynamics of the finite-time Lyapunov dimension is suggested. Various estimates of the finite-time Lya-
\end{abstract}

N. V. Kuznetsov $(\bowtie) \cdot$ G. A. Leonov · T. N. Mokaev

Saint-Petersburg State University, St. Petersburg, Russia e-mail: nkuznetsov239@gmail.com

N. V. Kuznetsov

Department of Mathematical Information Technology, University of Jyväskylä, Jyväskylä, Finland

A. Prasad

Department of Physics and Astrophysics, Delhi University, Delhi, India

M. D. Shrimali

Central University of Rajasthan, Ajmer, India punov dimension for the hidden attractor and hidden transient chaotic set in the case of multistability are given.

Keywords Hidden attractors · Perpetual points · Finite-time Lyapunov exponents · Adaptive algorithm for the computation of finite-time Lyapunov dimension

\section{Introduction}

One of the main tasks of the investigation of dynamical systems is the study of established (limiting) behaviour of the system after transient processes, i.e. the problem of localization and analysis of attractors (limited sets of the system states, which are reached by the system from close initial data after transient processes) [1-3]. While trivial attractors (stable equilibrium points) can be easily found analytically or numerically, the search of periodic and chaotic attractors can turn out to be a challenging problem (see, e.g. famous 16th Hilbert problem [4] on the number of coexisting periodic attractors in twodimensional polynomial systems, which was formulated in 1900 and is still unsolved, and its generalization for multidimensional systems with chaotic attractors [5]). For numerical localization of an attractor, one needs to choose an initial point in the basin of attraction and observe how the trajectory, starting from this initial point, after a transient process visualizes the attractor. Self-excited attractors, even coexisting in the case of multistability [6], can be revealed numerically by 
the integration of trajectories, started in small neighbourhoods of unstable equilibria, while hidden attractors have the basins of attraction, which are not connected with equilibria and are "hidden somewhere" in the phase space [7-10]. Remark that in numerical computation of trajectory over a finite-time interval, it is difficult to distinguish a sustained chaos from a transient chaos (a transient chaotic set in the phase space, which can persist for a long time) [11]. The search and visualization of hidden attractors and transient sets in the phase space are challenging tasks [12].

In this paper, we study hidden attractors and transient chaotic sets in the Rabinovich system. It is shown that the methods of numerical continuation and perpetual point are helpful for localization and understanding of hidden attractor in the Rabinovich system.

For the study of chaotic dynamics and dimension of attractors, the concept of the Lyapunov dimension [13] was found useful and became widely spread [1418]. Since only a finite time can be considered in the numerical analysis of dynamical system, in this paper we develop the concept of the finite-time Lyapunov dimension [19] and approaches to its reliable numerical computation. A new adaptive algorithm for the computation of finite-time Lyapunov dimension and exponents is used for studying the dynamics of the dimension. Various estimates of the finite-time Lyapunov dimension for the Rabinovich hidden attractor in the case of multistability are given.

\section{The Rabinovich system: interaction between waves in plasma}

Consider a system, studied in 1978 by Rabinovich [20] and Pikovski et al. [21],

$$
\begin{aligned}
& \dot{x}=h y-v_{1} x-y z, \\
& \dot{y}=h x-v_{2} y+x z, \\
& \dot{z}=-z+x y,
\end{aligned}
$$

describing the interaction of three resonantly coupled waves, two of which are parametrically excited. Here, the parameter $h$ is proportional to the pumping amplitude and the parameters $v_{1,2}$ are normalized dumping decrements.

After the linear transformation (see, e.g. [22]):

$$
\chi:(x, y, z) \rightarrow\left(v_{1} v_{2} h^{-1} y, v_{1} x, v_{1} v_{2} h^{-1} z\right),
$$

and time rescaling:

$t \rightarrow v_{1}^{-1} t$

we obtain a generalized Lorenz system:

$\dot{x}=-\sigma(x-y)-a y z$,

$\dot{y}=r x-y-x z$,

$\dot{z}=-b z+x y$,

where

$\sigma=v_{1}^{-1} v_{2}, b=v_{1}^{-1}, a=-v_{2}^{2} h^{-2}, r=v_{1}^{-1} v_{2}^{-1} h^{2}$.

System (4) with $a=0$ coincides with the classical Lorenz system [23]. As it is discussed in [22], system (4) can also be used to describe the following physical processes: the convective fluid motion inside rotating ellipsoid, the rotation of rigid body in viscous fluid, the gyrostat dynamics, the convection of horizontal layer of fluid making harmonic oscillations and the model of Kolmogorov's flow.

Note that since parameters $v_{1}, v_{2}, h$ are positive, the parameters $\sigma, b, r$ are positive and parameter $a$ is negative. From relation (5), we have:

$\sigma=-a r$

Further, we study system (4) under the assumption (6). If $r<1$, then system (4) has a unique equilibrium $S_{0}=(0,0,0)$, which is globally asymptotically Lyapunov stable (global attractor) [18,22]. If $r>1$, then system (4) has three equilibria: $\mathrm{S}_{0}=(0,0,0)$ and $\mathrm{S}_{ \pm}=\left( \pm x_{1}, \pm y_{1}, z_{1}\right)$, where

$x_{1}=\frac{\sigma b \sqrt{\xi}}{\sigma b+a \xi}, \quad y_{1}=\sqrt{\xi}, \quad z_{1}=\frac{\sigma \xi}{\sigma b+a \xi}$

and

$\xi=\frac{\sigma b}{2 a^{2}}\left[a(r-2)-\sigma+\sqrt{(\sigma-a r)^{2}+4 a \sigma}\right]$.

The stability of equilibria $S_{ \pm}$of system (4) depends on the parameters $r, a$ and $b$. Using the Routh-Hurwitz criterion, we obtain the following

Lemma 1 The equilibria $S_{ \pm}$of system (4) with parameters (5) are stable if and only if one of the following conditions holds: 
(i) $0 \leq a r+1<\frac{2 r}{r-\sqrt{r(r-1)}}$,

(ii) $a r+1<0, b>b_{c r}=\frac{4 a(r-1)(a r+1) \sqrt{r(r-1)}+(a r-1)^{3}}{(a r+1)^{2}-4 a r^{2}}$.

Proof The coefficients of the characteristic polynomial $\chi(x, y, z)=\lambda^{3}+p_{1}(x, y, z) \lambda^{2}+p_{2}(x, y, z) \lambda+$ $p_{3}(x, y, z)$ of the Jacobian matrix of system (4) at the point $(x, y, z)$ are the following:

$p_{1}(x, y, z)=b-a r+1$,

$p_{2}(x, y, z)=x^{2}+a y^{2}-a z^{2}-a r(b-r+1)+b$,

$p_{3}(x, y, z)=-a\left(2 x y z+r x^{2}-y^{2}+b z^{2}-b r(r-1)\right)$.

One can check that inequalities $p_{1}\left(x_{1}, y_{1}, z_{1}\right)>0$ and $p_{3}\left(x_{1}, y_{1}, z_{1}\right)>0$ are always valid. If $a r+1 \geq 0$, then $p_{1}\left(x_{1}, y_{1}, z_{1}\right) p_{2}\left(x_{1}, y_{1}, z_{1}\right)-p_{3}\left(x_{1}, y_{1}, z_{1}\right)>0$, and if condition (i) also holds, then $p_{2}\left(x_{1}, y_{1}, z_{1}\right)>0$.

If $a r+1<0$, then $p_{2}\left(x_{1}, y_{1}, z_{1}\right)>0$, and if condition (ii) also holds, then $p_{1}\left(x_{1}, y_{1}, z_{1}\right) p_{2}\left(x_{1}, y_{1}, z_{1}\right)-$ $p_{3}\left(x_{1}, y_{1}, z_{1}\right)>0$.

\section{Attractors and transient chaos}

Consider system (4) as an autonomous differential equation of general form:

$\dot{u}=f(u)$,

where $u=(x, y, z) \in \mathbb{R}^{3}$, and the continuously differentiable vector function $f: \mathbb{R}^{3} \rightarrow \mathbb{R}^{3}$ represents the right-hand side of system (4). Define by $u\left(t, u_{0}\right)$ a solution of (7) such that $u\left(0, u_{0}\right)=u_{0}$. For system (7), a bounded closed invariant set $K$ is

(i) a (local) attractor if it is a locally attractive set (i.e. $\lim _{t \rightarrow+\infty} \operatorname{dist}\left(K, u\left(t, u_{0}\right)\right)=0 \forall u_{0} \in K(\varepsilon)$, where $K(\varepsilon)$ is a certain $\varepsilon$-neighbourhood of set $K$ ),

(ii) a global attractor if it is a globally attractive set (i.e. $\left.\lim _{t \rightarrow+\infty} \operatorname{dist}\left(K, u\left(t, u_{0}\right)\right)=0 \forall u_{0} \in \mathbb{R}^{3}\right)$,

where $\operatorname{dist}(K, u)=\inf _{v \in K}\|v-u\|$ is the distance from the point $u \in \mathbb{R}^{3}$ to the set $K \subset \mathbb{R}^{3}$ (see, e.g. [9]). Since the whole phase space is a global attractor and any finite union of attractors is again an attractor, it is reasonable to consider only minimal global and local attractors, i.e. the smallest bounded closed invariant set possessing the property (ii) or (i).
Note that system (4) [or (7)] is dissipative in the sense that it possesses a bounded convex absorbing set $[9,22]$ :

$\mathcal{B}(r, a, b)=\left\{u \in \mathbb{R}^{3} \mid V(u) \leq \frac{b(\sigma+\delta r)^{2}}{2 c(a+\delta)}\right\}$,

where $V(u)=V(x, y, z)=x^{2}+\delta y^{2}+(a+$ $\delta)\left(z-\frac{\sigma+\delta r}{a+\delta}\right)^{2}, \delta$ is an arbitrary positive number such that $a+\delta>0$ and $c=\min \left(\sigma, 1, \frac{b}{2}\right)$. Thus, the solutions of (4) exist for $t \in[0,+\infty$ ) and system (4) possesses a global attractor $[9,24]$, which contains the set of all equilibria.

Computational errors (caused by a finite precision arithmetic and numerical integration of differential equations) and sensitivity to initial data allow one to get a visualization of chaotic attractor by one pseudotrajectory computed for a sufficiently large time interval. One needs to choose an initial point in the basin of attraction of the attractor and observe how the trajectory, starting from this initial point, after a transient process visualizes the attractor. Thus, from a computational point of view, it is natural to suggest the following classification of attractors, based on the simplicity of finding the basins of attraction in the phase space.

Definition $1[7,9,10,25]$ An attractor is called a selfexcited attractor if its basin of attraction intersects with any open neighbourhood of an equilibrium; otherwise it is called a hidden attractor.

For a self-excited attractor, its basin of attraction is connected with an unstable equilibrium and, therefore, self-excited attractors can be found numerically by the standard computational procedure in which after a transient process, a trajectory, starting in a neighbourhood of an unstable equilibrium, is attracted to the state of oscillation and then traces it; then, the computations are being performed for a grid of points in vicinity of the state of oscillation to explore the basin of attraction and improve the visualization of the attractor. Thus, self-excited attractors can be easily visualized (e.g. the classical Lorenz and Hénon attractors are self-excited with respect to all existing equilibria and can be easily visualized by a trajectory from their vicinities).

For a hidden attractor, its basin of attraction is not connected with equilibria and, thus, the search and visualization of hidden attractors in the phase space 
may be a challenging task. Hidden attractors are attractors in the systems without equilibria (see, e.g. rotating electromechanical systems with Sommerfeld effect (1902) [26,27]), and in the systems with only one stable equilibrium (see, e.g. counterexamples [7,28] to Aizerman's (1949) and Kalman's (1957) conjectures on the monostability of nonlinear control systems $[29,30])$. One of the first related problems is the second part of 16th Hilbert problem [4] on the number and mutual disposition of limit cycles in two-dimensional polynomial systems, where nested limit cycles (a special case of multistability and coexistence of periodic attractors) exhibit hidden periodic attractors (see, e.g. [7,31,32]). The classification of attractors as being hidden or selfexcited was introduced by Leonov and Kuznetsov in connection with the discovery of the first hidden Chua attractor [25,33-38] and has captured much attention of scientists from around the world (see, e.g. [39-68]).

Since in numerical computation of trajectory over a finite-time interval, it is difficult to distinguish a sustained chaos from a transient chaos (a transient chaotic set in the phase space, which can nevertheless persist for a long time) [11,69], a similar classification can be introduced for the transient chaotic sets.

Definition 2 [70,71] A transient chaotic set is called a hidden transient chaotic set if it does not involve and attract trajectories from a small neighbourhood of equilibria; otherwise, it is called self-excited.

In order to distinguish an attracting chaotic set (attractor) from a transient chaotic set in numerical experiments, one can consider a grid of points in a small neighbourhood of the set and check the attraction of corresponding trajectories towards the set.

For system (1) with parameters $v_{1}=1, v_{2}=4$, and increasing $h$, it is possible to observe [21] the classical scenario of transition to chaos (via homoclinic and subcritical Andronov-Hopf bifurcations) similar to the scenario in the Lorenz system. For $4.84 \lesssim h \lesssim 13.4$ in system (1), there is a self-excited chaotic attractor (see e.g. Fig. 1), which coexists with two stable equilibria. The same scenario can be obtained for system (4) when parameters $b>0$ and $a<0$ are fixed and $r$ is increasing. Besides self-excited chaotic attractors, a hidden attractor was found $[71,72]$ in the system. Note that in $[9,73]$, system (4) with $a>0$ was studied and a hidden attractor was also found numerically.

Further, we localize a hidden chaotic attractor in system (4) with $a<0$ by the numerical continuation

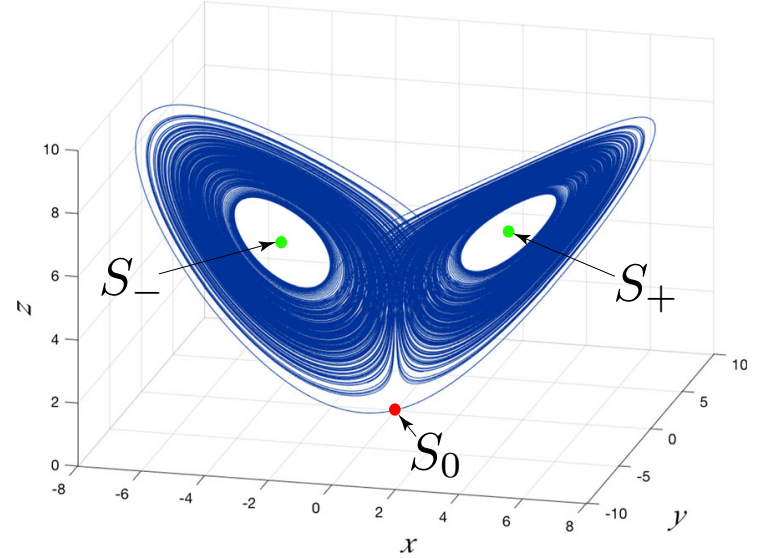

Fig. 1 Multistability in the Rabinovich system (4) with the classical values of parameters $v_{1}=1, v_{2}=4, h=4.92$ from [21]: coexistence of three local attractors-two stable equilibria $S_{ \pm}$ and a chaotic self-excited attractor (self-excited with respect to the unstable zero equilibrium $S_{0}$ )

method starting from a self-excited chaotic attractor. We change parameters, considered in [72], in such a way that the chaotic set is located not too close to the unstable zero equilibrium to avoid a situation, when numerically integrated trajectory oscillates for a long time and then falls on the unstable manifold of unstable zero equilibrium, leaves the chaotic set and tends to one of the stable equilibria.

\subsection{Localization via numerical continuation method}

One of the effective methods for numerical localization of hidden attractors in multidimensional dynamical systems is based on the homotopy and numerical continuation method (NCM). It is based on the assumption that the position of the attractor changes continuously with the parameters changing. The idea is to construct a sequence of similar systems such that for the first (starting) system, the initial point for numerical computation of oscillating solution (starting attractor) can be obtained analytically, for example, it is often possible to consider the starting system with a self-excited starting attractor; then, the transformation of this starting attractor in the phase space is tracked numerically while passing from one system to another; the last system corresponds to the system in which a hidden attractor is searched.

For the study of the scenario of transition to chaos, we consider system (7) with $f(u)=f(u, \lambda)$, where 
$\lambda \in \Lambda \subset \mathbb{R}^{d}$ is a vector of parameters, whose variation in the parameter space $\Lambda$ determines the scenario. Let $\lambda_{\text {end }} \in \Lambda$ define a point corresponding to the system, where a hidden attractor is searched. Choose a point $\lambda_{\text {begin }} \in \Lambda$ such that we can analytically or numerically localize a certain nontrivial (oscillating) attractor $\mathcal{A}^{1}$ in system (7) with $\lambda=\lambda_{\text {begin }}$ (e.g. one can consider a self-excited attractor, defined by a trajectory $u^{1}(t)$ numerically integrated on a sufficiently large time interval $t \in[0, T]$ with the initial point $u^{1}(0)$ in the vicinity of an unstable equilibrium). Consider a path ${ }^{1}$ in the parameter space $\Lambda$, i.e. a continuous function $\gamma:[0,1] \rightarrow \Lambda$ for which $\gamma(0)=\lambda_{\text {begin }}$ and $\gamma(1)=\lambda_{\text {end }}$, and a sequence of points $\left\{\lambda^{j}\right\}_{j=1}^{k}$ on the path, where $\lambda^{1}=\lambda_{\text {begin }}, \lambda^{k}=\lambda_{\text {end }}$, such that the distance between $\lambda^{j}$ and $\lambda^{j+1}$ is sufficiently small. On each next step of the procedure, the initial point for a trajectory to be integrated is chosen as the last point of the trajectory integrated on the previous step: $u^{j+1}(0)=u^{j}(T)$. Following this procedure and sequentially increasing $j$, two alternatives are possible: the points of $\mathcal{A}^{j}$ are in the basin of attraction of attractor $\mathcal{A}^{j+1}$, or while passing from system (7) with $\lambda=\lambda^{j}$ to system (7) with $\lambda=\lambda^{j+1}$, a loss of stability bifurcation is observed and attractor $\mathcal{A}^{j}$ vanishes. If under changing $\lambda$ from $\lambda_{\text {begin }}$ to $\lambda_{\text {end }}$, there is no loss of stability bifurcation of the considered attractors, then a hidden attractor for $\lambda^{k}=\lambda_{\text {end }}$ (at the end of the procedure) is localized.

\subsection{Localization using perpetual points}

The equilibrium points of a dynamical system are the ones at which the velocity and acceleration of the system simultaneously become zero. In this section, we show that there are points, termed as perpetual points [74], which may help to visualize hidden attractors.

For system (7), the equilibrium points $u_{\text {ep }}$ are defined by the equation $\dot{u}=f\left(u_{\mathrm{ep}}\right)=0$. Consider a derivative of system (7) with respect to time:

$\ddot{u}=J(u) f(u)=g(u)$,

where $J(u)=\left[\frac{\partial f_{i}(u)}{\partial u_{j}}\right]_{i, j=1}^{3}$ is the Jacobian matrix. Here, $g(u)$ may be termed as an acceleration vector.

1 In the simplest case, when $d=1$, the path is a line segment.
System (9) shows the variation of acceleration in the phase space.

Similar to the equilibrium points estimation, where we set the velocity vector to zero, we can also get a set of points, where $\ddot{u}=g\left(u_{\mathrm{pp}}\right)=0$ in (9), i.e. the points corresponding to the zero acceleration. At these points, the velocity $\dot{u}$ may be either zero or nonzero. This set includes the equilibrium points $u_{\text {ep }}$ with zero velocity as well as a subset of points with nonzero velocity. These nonzero velocity points $u_{\mathrm{pp}}$ are termed as perpetual points [12,74-76].

Lemma 2 Perpetual points $S_{\mathrm{pp}}=\left(x_{\mathrm{pp}}, y_{\mathrm{pp}}, z_{\mathrm{pp}}\right)$ of system (4) can be derived from the following system

$$
\left\{\begin{array}{c}
\left(x_{\mathrm{pp}}^{2}-a z_{\mathrm{pp}}^{2}+a r^{2}-1\right)\left(-y_{\mathrm{pp}}^{2}+z_{\mathrm{pp}}^{2}+r^{2}(a-1)\right) \\
=r^{2}(a r-1)^{2}-z_{\mathrm{pp}}^{2}(a r-b-1)^{2}, \\
2 x_{\mathrm{pp}} y_{\mathrm{pp}} z_{\mathrm{pp}}+r x_{\mathrm{pp}}^{2}-y_{\mathrm{pp}}^{2}+b z_{\mathrm{pp}}^{2}-b r(r-1)=0, \\
z_{\mathrm{pp}}=\frac{r x_{\mathrm{pp}}^{2}+(a r-b-1) x_{\mathrm{pp}} y_{\mathrm{pp}}-a r y_{\mathrm{pp}}^{2}}{x_{\mathrm{pp}}^{2}+a y_{\mathrm{pp}}^{2}-b^{2}} .
\end{array}\right.
$$

The reason why perpetual points may lead to hidden states [perpetual point method (PPM)] is still discussed (see, e.g. $[77,78]$ ).

\section{Hidden attractor in the Rabinovich system}

Next, we apply the NCM for localization of a hidden attractor in the Rabinovich system (4) and check whether the attractor can be also localized using PPM.

In this experiment, we fix parameter $r$ and, using condition (ii) of Lemma 1, define parameters $a=$ $-\frac{1}{r}-\varepsilon_{1}$ and $b=b_{c r}-\varepsilon_{2}$. For $r=100, \varepsilon_{1}=10^{-3}$, $\varepsilon_{2}=10^{-2}$, we obtain $a=a_{0} \equiv-1.1 \times 10^{-2}$, $b=b_{0} \equiv 6.7454 \times 10^{-2}$ and take $P_{0}\left(a_{0}, b_{0}\right)$ as the initial point of line segment on the plane $(a, b)$. The eigenvalues of the Jacobian matrix at the equilibria $S_{0}, S_{ \pm}$of system (4) for these parameters are the following:

$S_{0}: 9.4382, \quad-0.0675, \quad-11.5382$,

$S_{ \pm}: \quad 0.0037 \pm 3.6756 i, \quad-2.1749$.

Consider on the plane $(a, b)$ a line segment, intersecting a boundary of stability domain of the equilib- 


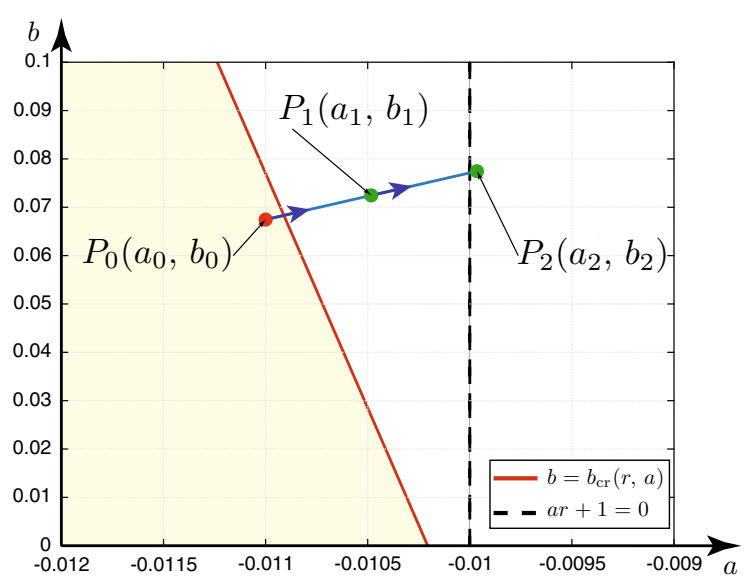

Fig. 2 Path $P_{0}\left(a_{0}, b_{0}\right) \rightarrow P_{1}\left(a_{1}, b_{1}\right) \rightarrow P_{2}\left(a_{2}, b_{2}\right)$ in parameters plane $(a, b)$ for the localization of hidden attractor in system (4) with $r=100$. Here, $a_{0}=-1.1 \times 10^{-2}, b_{0}=$ $6.7454 \times 10^{-2}, a_{1}=-1.049 \times 10^{-2}, b_{1}=7.2454 \times 10^{-2}$, $a_{2}=-9.965 \times 10^{-3}, b_{2}=7.7454 \times 10^{-3} ;(\bullet) P_{0}\left(a_{0}, b_{0}\right)$ : self-excited attractor with respect to $S_{0}, S_{ \pm} ;(\bullet) P_{1}\left(a_{1}, b_{1}\right)$ : selfexcited attractor with respect to $S_{0} ;(\bullet) P_{2}\left(a_{2}, b_{2}\right)$ : hidden attractor. Stability domain is defined using Lemma 1

ria $S_{ \pm}$with the final point $P_{2}\left(a_{2}, b_{2}\right)$, where $a_{2}=$ $a_{0}+1.035 \times 10^{-3}=-9.965 \times 10^{-3}, b_{2}=b_{0}+\varepsilon_{2}=$ $7.7454 \times 10^{-2}$, i.e. the equilibrium $S_{0}$ remains saddle and the equilibria $S_{ \pm}$become stable focus-nodes

$$
\begin{array}{ccc}
S_{0}: & 8.9842, \quad-0.0775, & -10.9807 \\
S_{ \pm}: & -0.0401 \pm 3.9152 i, & -1.9937
\end{array}
$$

The initial point $P_{0}\left(a_{0}, b_{0}\right)$ corresponds to the parameters for which in system (4), there exists a selfexcited attractor. Then for the considered line segment, a sufficiently small partition step is chosen, and at each iteration step of the procedure, an attractor in the phase space of system (4) is computed. The last computed point at each step is used as the initial point for the computation at the next step. In this experiment, we use NCM with 3 steps on the path $P_{0}\left(a_{0}, b_{0}\right) \rightarrow$ $P_{1}\left(a_{1}, b_{1}\right) \rightarrow P_{2}\left(a_{2}, b_{2}\right)$, with $a_{1}=\frac{1}{2}\left(a_{0}+a_{2}\right)$, $b_{1}=\frac{1}{2}\left(b_{0}+b_{2}\right)$ (see Fig. 2). At the first step, we have self-excited attractor with respect to unstable equilibria $S_{0}$ and $S_{ \pm}$; at the second step, the equilibria $S_{ \pm}$become stable but the attractor remains self-excited with respect to equilibrium $S_{0}$; at the third step, it is possible to visualize a hidden attractor of system (4) (see Fig. 3).

Using Lemma 2 for parameters $r=100, a=$ $-9.965 \times 10^{-3}, b=7.7454 \times 10^{-2}$, we obtain one perpetual point $S_{\mathrm{pp}}=(-0.2385,49.1403$, - 101.4613), which allows one to localize a hidden attractor (see Fig. 4). Hence, here both NCM and PPM allow one to find this hidden attractor.

Around equilibrium $S_{0}$, we choose a small spherical vicinity of radius $\delta$ (in our experiments, we check $\delta \in[0.1,0.5])$ and take $N$ random initial points on it (in our experiment, $N=4000$ ). Using MATLAB, we integrate system (4) with these initial points in order to explore the obtained trajectories. We repeat this procedure several times in order to get different initial points for trajectories on the sphere. We get the following results: all the obtained trajectories attract to either the stable equilibrium $S_{+}$or the equilibrium $S_{-}$ and do not tend to the attractor. This gives us a reason to classify the chaotic attractor, obtained in system (4), as the hidden one.

Remark that there exist hidden chaotic sets in the Rabinovich system, which cannot be localized by PPM. For example, for parameters $r=6.8, a=-0.5, b \in$ $[0.99,1][72]$, the hidden attractor obtained by NCM is not localizable via PPM (see Fig. 5).

\section{Computation of the Lyapunov dimension}

5.1 Finite-time and limit values of the Lyapunov dimension and Lyapunov exponents

The Lyapunov exponents [2] and Lyapunov dimension [13] are widely used for the study of attractors (see, e.g. [14-18]). Nowadays, various approaches to the definition of Lyapunov dimension are used. Since in numerical experiments we can consider only finite time, in this paper we develop the concept of finite-time Lyapunov dimension [19] and an approach to its reliable numerical computation. This approach is inspirited by the works of Douady and Oesterlé [79], Hunt [80], and Rabinovich et al. [81].

For a fixed $t \geq 0$ let us consider the map $\varphi^{t}: \mathbb{R}^{3} \rightarrow$ $\mathbb{R}^{3}$ defined by the shift operator along the solutions of system (7): $\varphi^{t}\left(u_{0}\right)=u\left(t, u_{0}\right), u_{0} \in \mathbb{R}^{3}$. Since system (7) possesses an absorbing set [see (8)], the existence and uniqueness of solutions of system (7) for $t \in[0,+\infty)$ take place and, therefore, the system generates a dynamical system $\left\{\varphi^{t}\right\}_{t \geq 0}$. Let a nonempty closed bounded set $K \subset \mathbb{R}^{3}$ be invariant with respect to the dynamical system $\left\{\varphi^{t}\right\}_{t \geq 0}$, i.e. $\varphi^{t}(K)=K$ for all $t \geq 0$ (e.g. $K$ is an attractor). Further, we use 


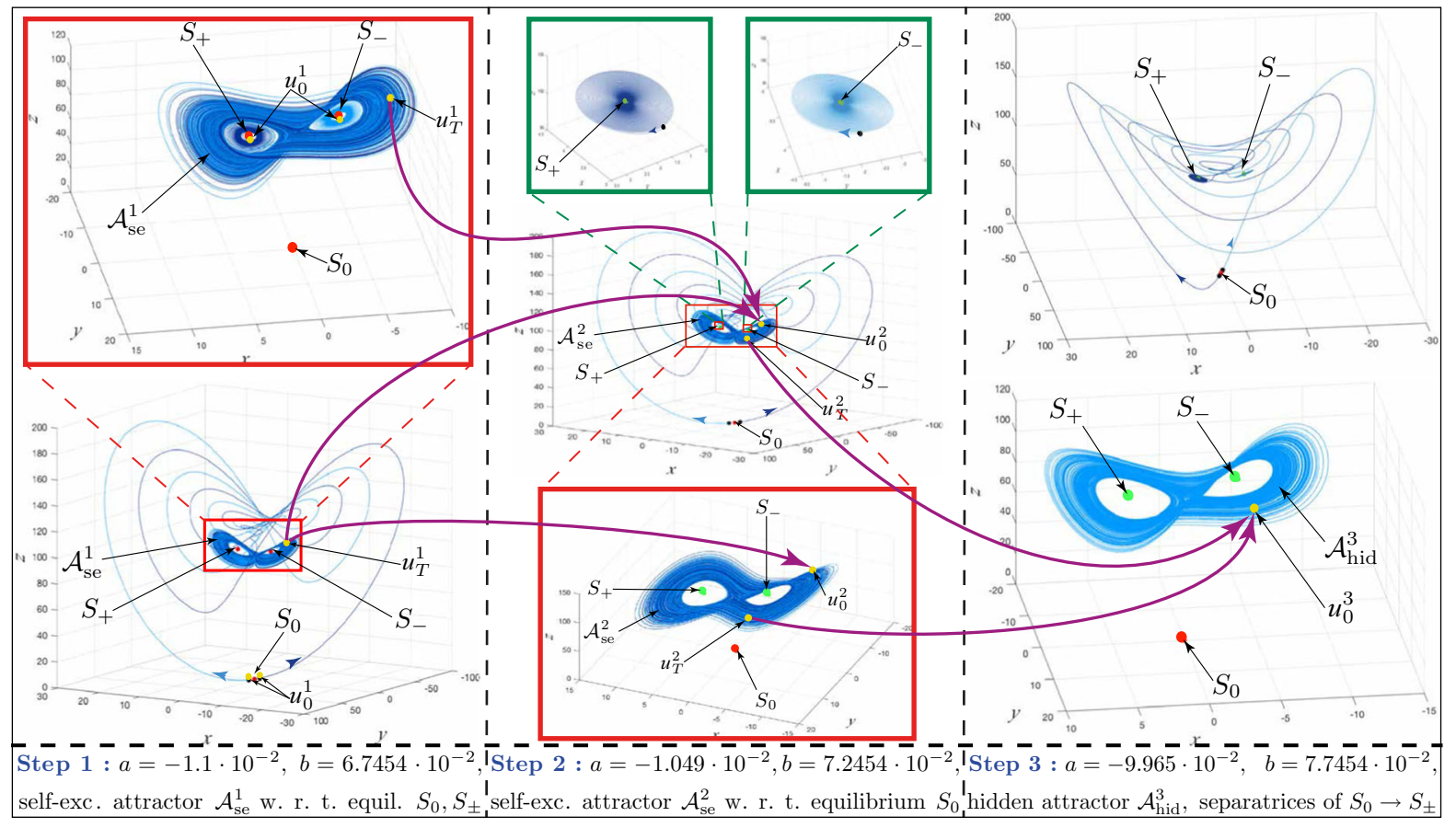

Fig. 3 Localization, by NCM, of a hidden attractor in system (4) with $r=100, a=-9.965 \times 10^{-3}, b=7.7454 \times 10^{-2}$. Trajectories $u^{i}(t)=\left(x^{i}(t), y^{i}(t), z^{i}(t)\right.$ (blue) are defined on the time interval $\left[0, T=10^{3}\right]$, and initial point (yellow) on

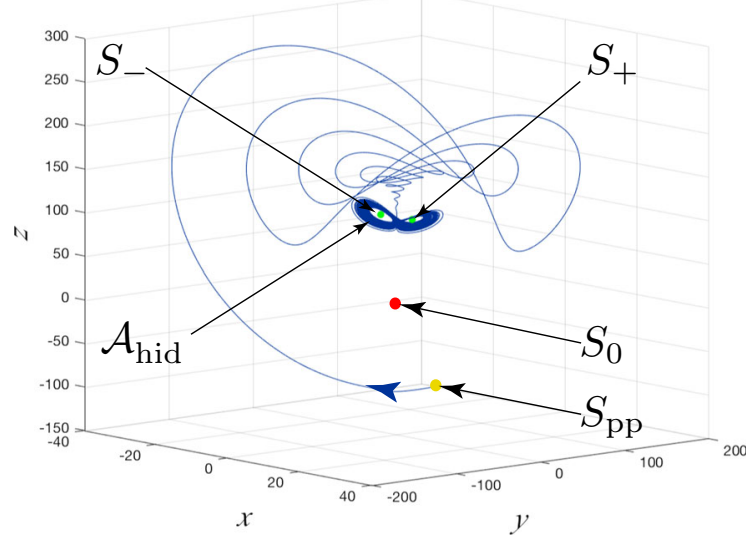

Fig. 4 Localization of hidden attractor in system (4) with $r=$ $100, a=-9.965 \times 10^{-3}, b=7.7454 \times 10^{-2}$ from the perpetual point $S_{\mathrm{pp}}=(-0.2385,49.1403,-101.4613)$

compact notations for the finite-time local Lyapunov dimension: $\operatorname{dim}_{\mathrm{L}}(t, u)=\operatorname{dim}_{\mathrm{L}}\left(\varphi^{t}, u\right)$, the finite-time Lyapunov dimension: $\operatorname{dim}_{\mathrm{L}}(t, K)=\operatorname{dim}_{\mathrm{L}}\left(\varphi^{t}, K\right)$ and for the Lyapunov dimension (or the Lyapunov dimen- $(i+1)$-th iteration is defined as $u_{0}^{i+1}:=u_{T}^{i}$ (violet arrows), where $u_{T}^{i}=u^{i}(T)$ is a final point (yellow). (Color figure online)

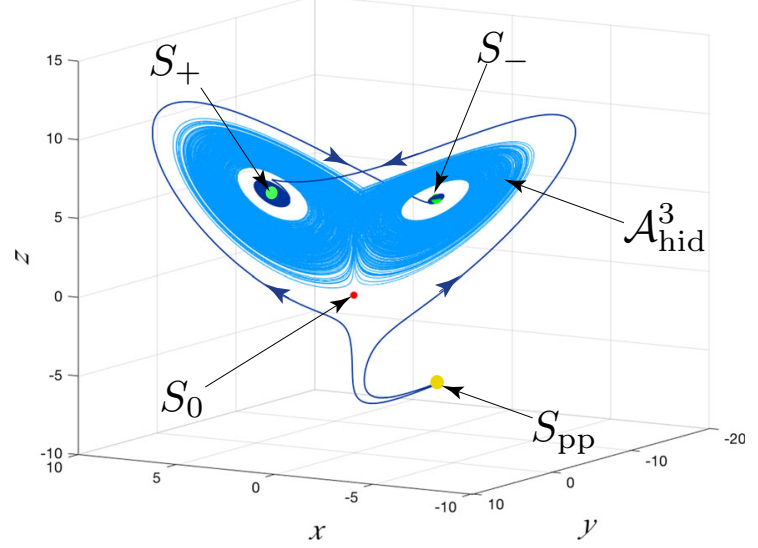

Fig. 5 Hidden attractor in system (4) with $r=6.8, a=-0.5$, $b=0.99$, which can be localized via NCM (initial point $u_{0}=$ $(-0.1629,-0.2154,1.9553))$, but cannot be localized from the perpetual point $S_{\mathrm{pp}}=(0.7431,-12.6109,-7.4424)$ (yellow). (Color figure online)

sion of dynamical system $\left\{\varphi^{t}\right\}_{t \geq 0}$ on invariant set $\left.K\right)$ : $\operatorname{dim}_{\mathrm{L}} K=\operatorname{dim}_{\mathrm{L}}\left(\left\{\varphi^{t}\right\}_{t \geq 0}, K\right)$.

Consider linearization of system (7) along the solution $\varphi^{t}(u)$ : 
$\dot{v}=J\left(\varphi^{t}(u)\right) v, \quad J(u)=D f(u)$,

where $J(u)$ is the $3 \times 3$ Jacobian matrix, the elements of which are continuous functions of $u$, and suppose that det $J(u) \neq 0 \quad \forall u \in \mathbb{R}^{3}$. Consider a fundamental matrix of solutions of linear system (11), $D \varphi^{t}(u)$, such that $D \varphi^{0}(u)=I$, where $I$ is a unit $3 \times 3$ matrix. Denote by $\sigma_{i}(t, u)=\sigma_{i}\left(D \varphi^{t}(u)\right), i=1,2,3$, the singular values of $D \varphi^{t}(u)$ (i.e. $\sigma_{i}(t, u)>0$ and $\sigma_{i}(t, u)^{2}$ are the eigenvalues of the symmetric matrix $D \varphi^{t}(u)^{*} D \varphi^{t}(u)$ with respect to their algebraic multiplicity) ${ }^{2}$ ordered so that $\sigma_{1}(t, u) \geq \sigma_{2}(t, u) \geq \sigma_{3}(t, u)>0$ for any $u \in \mathbb{R}^{3}$ and $t>0$.

A singular value function of order $d \in[0,3]$ is defined as

$$
\begin{aligned}
& \omega_{d}\left(D \varphi^{t}(u)\right)=\sigma_{1}(t, u) \cdots \sigma_{\lfloor d\rfloor}(t, u) \sigma_{\lfloor d\rfloor+1}(t, u)^{d-\lfloor d\rfloor}, \\
& \omega_{0}\left(D \varphi^{t}(u)\right)=1, \omega_{3}\left(D \varphi^{t}(u)\right)=\sigma_{1}(t, u) \sigma_{2}(t, u) \sigma_{3}(t, u),
\end{aligned}
$$

where $\lfloor d\rfloor$ is the largest integer less or equal to $d$. For a certain moment of time $t \geq 0$, the finite-time local Lyapunov dimension at the point $u$ is defined as [19]

$\operatorname{dim}_{\mathrm{L}}(t, u)=\max \left\{d \in[0,3]: \omega_{d}\left(D \varphi^{t}(u)\right) \geq 1\right\}$

and the finite-time Lyapunov dimension of $K$ is defined as

$\operatorname{dim}_{\mathrm{L}}(t, K)=\sup _{u \in K} \operatorname{dim}_{\mathrm{L}}(t, u)$.

The Douady-Oesterlé theorem [79] implies that for any fixed $t \geq 0$, the Lyapunov dimension of the map $\varphi^{t}$ with respect to a closed bounded invariant set $K$, defined by (13), is an upper estimate of the Hausdorff dimension of the set $K: \operatorname{dim}_{\mathrm{H}} K \leq \operatorname{dim}_{\mathrm{L}}(t, K)$.

For the estimation of the Hausdorff dimension of invariant closed bounded set $K$, one can use the map $\varphi^{t}$ with any time $t$ (e.g. $t=0$ leads to the trivial estimate $\operatorname{dim}_{\mathrm{H}} K \leq 3$ ) and, thus, the best estimation is $\operatorname{dim}_{\mathrm{H}} K \leq \inf _{t \geq 0} \operatorname{dim}_{\mathrm{L}}(t, K)$. The following property

$\inf _{t \geq 0} \sup _{u \in K} \operatorname{dim}_{\mathrm{L}}(t, u)=\liminf _{t \rightarrow+\infty} \sup _{u \in K} \operatorname{dim}_{\mathrm{L}}(t, u)$

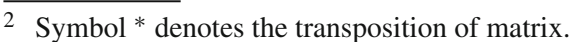

allows one to introduce the Lyapunov dimension of $K$ as [19]

$\operatorname{dim}_{\mathrm{L}} K=\liminf _{t \rightarrow+\infty} \sup _{u \in K} \operatorname{dim}_{\mathrm{L}}(t, u)$

and get an upper estimation of the Hausdorff dimension:

$\operatorname{dim}_{\mathrm{H}} K \leq \operatorname{dim}_{\mathrm{L}} K$.

Recall that a set $K$ with noninteger Hausdorff dimension is referred to as a fractal set [15] and, when such set $K$ is an attractor, it is called a strange attractor [82,83].

Consider a set of finite-time Lyapunov exponents ${ }^{3}$ at the point $u$ :

$\mathrm{LE}_{i}(t, u)=\frac{1}{t} \ln \sigma_{i}(t, u), t>0, \quad i=1,2,3$.

Here, the set $\left\{\operatorname{LE}_{i}(t, u)\right\}_{i=1}^{3}$ is ordered by decreasing (i.e. $\operatorname{LE}_{1}(t, u) \geq \operatorname{LE}_{2}(t, u) \geq \operatorname{LE}_{3}(t, u)$ for all $t>0)$. Then for $j(t, u)=\left\lfloor\operatorname{dim}_{\mathrm{L}}(t, u)\right\rfloor<3$ and $s(t, u)=\operatorname{dim}_{\mathrm{L}}(t, u)-\left\lfloor\operatorname{dim}_{\mathrm{L}}(t, u)\right\rfloor$, we have $0=$ $\frac{1}{t} \ln \left(\omega_{j(t, u)+s(t, u)}\left(D \varphi^{t}(u)\right)\right)=\sum_{i=1}^{j(t, u)} \mathrm{LE}_{i}(t, u)+$ $s(t, u) \operatorname{LE}_{j(t, u)+1}(t, u)$ and $j(t, u)=\max$ $\left\{m: \sum_{i=1}^{m} \operatorname{LE}_{i}(t, u) \geq 0\right\}$. Thus, we get an analog of the Kaplan-Yorke formula [13] with respect to the set of finite-time Lyapunov exponents $\left\{\operatorname{LE}_{i}(t, u)\right\}_{i=1}^{3}$ [19]:

$\left.d_{\mathrm{L}}^{\mathrm{KY}}\left(\operatorname{LE}_{i}(t, u)\right\}_{i=1}^{3}\right)=j(t, u)+\frac{\mathrm{LE}_{1}(t, u)+\cdots+\mathrm{LE}_{j(t, u)}(t, u)}{\left|\mathrm{LE}_{j(t, u)+1}(t, u)\right|}$,

which gives the finite-time local Lyapunov dimension: $\operatorname{dim}_{\mathrm{L}}(t, u)=d_{\mathrm{L}}^{\mathrm{KY}}\left(\left\{\mathrm{LE}_{i}(t, u)\right\}_{i=1}^{3}\right)$.

Thus, in the above approach, the use of Kaplan-Yorke formula (17) with the finite-time Lyapunov exponents $\left\{\mathrm{LE}_{i}(t, u)\right\}_{i=1}^{3}$ is rigorously justified by the DouadyOesterlé theorem.

Note that the Lyapunov dimension is invariant under Lipschitz diffeomorphisms $[19,86]$, i.e. if the dynamical system $\left\{\varphi^{t}\right\}_{t \geq 0}$ and closed bounded invariant set

\footnotetext{
3 The Lyapunov exponents, LEs (see, e.g. [84]) characterize the rates of exponential growth of the singular values of fundamental matrix of the linearized system. The singular values correspond to the semiaxes of $n$-dimensional ellipsoid, which is the image of the unit sphere with respect to the linearized system. See [85] for various related notions.
} 
$K$ under a smooth change of coordinates $w=\chi(u)$ are transformed into the dynamical system $\left\{\varphi_{\chi}^{t}\right\}_{t \geq 0}$ and closed bounded invariant set $\chi(K)$, respectively, then $\operatorname{dim}_{\mathrm{L}}\left(\left\{\varphi^{t}\right\}_{t \geq 0}, K\right)=\operatorname{dim}_{\mathrm{L}}\left(\left\{\varphi_{\chi}^{t}\right\}_{t \geq 0}, \chi(K)\right)$. Also the Lyapunov dimension is invariant under positive time rescaling $t \rightarrow a t, a>0$.

\subsection{Adaptive algorithm for the computation of the finite-time Lyapunov dimension and exponents}

Applying the statistical physics approach and assuming the ergodicity (see, e.g. $[13,87-89]$ ), the Lyapunov dimension of attractor $\operatorname{dim}_{\mathrm{L}} K$ is often estimated by the local Lyapunov dimension $\operatorname{dim}_{\mathrm{L}}\left(t, u_{0}\right)$, corresponding to a "typical" trajectory, which belongs to the attractor: $\left\{u\left(t, u_{0}\right), t \geq 0\right\}, u_{0} \in K$, and its limit value $\lim _{t \rightarrow+\infty} \operatorname{dim}_{\mathrm{L}}\left(t, u_{0}\right)$. However, from a practical point of view, the rigorous verification of ergodicity is a challenging task $[84,90]$ and hardly it can effectively be done in a general case (see, e.g. discussions in [91], [92, p. 118], [93], [94, p. 9], [95, p. 19], and the works $[96,97]$ on the Perron effects of the largest Lyapunov exponent sign reversals). Examples of the rigorous use of the ergodic theory for the computation of the Lyapunov exponents and dimension can be found, for example, in [87,98-100]. In the work by Yorke et al. [88, p. 190], the exact limit values of finitetime Lyapunov exponents $\left\{\lim _{t \rightarrow+\infty} \operatorname{LE}_{i}\left(t, u_{0}\right)\right\}_{i}^{3}=$ $\left\{\operatorname{LE}_{i}\left(u_{0}\right)\right\}$, if they exist and are the same for all $u_{0} \in \mathbb{R}^{3}$, i.e.

$$
\lim _{t \rightarrow+\infty} \operatorname{LE}_{i}\left(t, u_{0}\right)=\operatorname{LE}_{i}\left(u_{0}\right) \equiv \mathrm{LE}_{i} \quad \forall u_{0} \in \mathbb{R}^{3}, i=1,2,3,
$$

are called the absolute ones, and it is noted that the absolute Lyapunov exponents rarely exist ${ }^{4}$ (in this case, one also has $\operatorname{dim}_{\mathrm{L}} K=d_{\mathrm{L}}^{\mathrm{KY}}\left(\left\{\mathrm{LE}_{i}\right\}_{1}^{3}\right)=j+$ $\left.\frac{\mathrm{LE}_{1}+\cdots+\mathrm{LE}_{j}}{\left|\mathrm{LE}_{j+1}\right|}\right)$

\footnotetext{
4 For example, let us consider the Hénon map with parameters $a=0.97$ and $b=0.466$. In this case [101] for initial points $u_{0}^{1}=$ $(-1.287342,-1.287401)$ and $u_{0}^{2}=(0.840503,0.704249)$ after a transient process during $\left[0, T_{\text {trans }}=10^{5}\right]$, we get points $u_{0}^{\mathrm{a} 1}$ and $u_{0}^{\mathrm{a} 2}$, respectively, and compute finite-time Lyapunov exponents and finite-time local Lyapunov dimension for the time interval $\left[0, T=10^{4}\right]$ by the adaptive algorithm with $\delta=10^{-8}: \operatorname{LE}_{1}\left(T, u_{0}^{\mathrm{a} 1}\right) \approx 0.01198, \operatorname{dim}_{\mathrm{L}}\left(T, u_{0}^{\mathrm{a} 1}\right) \approx 1.01545$ and $\operatorname{LE}_{1}\left(T, u_{0}^{\mathrm{a} 2}\right) \approx 0.01405, \operatorname{dim}_{\mathrm{L}}\left(T, u_{0}^{\mathrm{a} 2}\right) \approx 1.01807$, the Lyapunov dimensions of two stationary points are 1.42787 and 1.56938 and some trajectories $u\left(t, u_{0}\right)$ and corresponding $\operatorname{LE}_{1}\left(t, u_{0}\right)$ tend to infinity. In general, the important question [5] is whether the system possesses coexisting attractors and how to find them all?
}

Note also that even if a numerical approximation (visualization) $\widetilde{K}$ of the attractor $K$ is obtained, it is not straightforward how to get a point on the attractor itself: $u_{0} \in K$. Thus, an easy way to get reliable estimation of the Lyapunov dimension of attractor $K$ is to localize the attractor $K \subset K^{\varepsilon}$, to consider a grid of points $K_{\text {grid }}^{\varepsilon}$ on $K^{\varepsilon}$, and to find the maximum of the corresponding finite-time local Lyapunov dimensions for a certain time $t=T: \max _{u \in K_{\text {grid }}^{\varepsilon}} \operatorname{dim}_{\mathrm{L}}\left(\varphi^{T}, u\right)=$ $\max _{u \in K_{\text {grid }}^{\varepsilon}} j(T, u)+\frac{\operatorname{LE}_{1}(T, u)+\cdots+\operatorname{LE}_{j(T, u)}(T, u)}{\left|\operatorname{LE}_{j(T, u)+1}(T, u)\right|}$.

Concerning the time $T$, remark that while the time series obtained from a physical experiment are assumed to be reliable on the whole considered time interval, the time series produced by the integration of mathematical dynamical model (7) can be reliable on a limited time interval only ${ }^{5}$ due to computational errors (caused by finite precision arithmetic and numerical integration of ODE). Thus, in general, the closeness of the real trajectory $u\left(t, u_{0}\right)$ and the corresponding pseudotrajectory $\tilde{u}\left(t, u_{0}\right)$ calculated numerically can be guaranteed on a limited short time interval only. For the numerical visualization of a chaotic attractor, the computation of a pseudo-trajectory on a longer time interval often allows one to obtain a more complete visualization of the attractor (pseudo-attractor) due to computational errors and sensitivity to initial data. However, for two different long-time pseudo-trajectories $\tilde{u}\left(t, u_{0}^{1}\right)$ and $\tilde{u}\left(t, u_{0}^{2}\right)$ visualizing the same attractor, the corresponding finite-time LEs can be, within the considered error, similar due to averaging over time [see (16)] and similar sets of points $\left\{\tilde{u}\left(t, u_{0}^{1}\right)\right\}_{t \geq 0}$ and $\left\{\tilde{u}\left(t, u_{0}^{2}\right)\right\}_{t \geq 0}$. At the same time, the corresponding real trajectories $u\left(t, u_{0}^{1,2}\right)$ may have different LEs (e.g. $u_{0}$ may correspond to an unstable periodic trajectory $u\left(t, u_{0}\right)$, which is embedded in the attractor and does not allow one to visualize it). Here, one may recall the question [105, p. 98] (known as Eden conjecture) whether the supremum of the local Lyapunov dimensions is achieved on a stationary point or an unstable periodic orbit embedded in

\footnotetext{
5 In $[102,103]$ for the Lorenz system the time interval of reliable computation with 16 significant digits and error $10^{-4}$ is estimated as $[0,36]$, with error $10^{-8}$ is estimated as $[0,26]$, and reliable computation for a longer time interval, e.g. [0, 10,000] in [104], is a challenging task. Also if $u_{0}$ belongs to a transient chaotic set, then $u\left(t, u_{0}\right)$ may have positive finite-time Lyapunov exponent on a very large time interval, e.g. $[0,15,000]$, but finally $u\left(t, u_{0}\right)$ converges to a stable stationary point as $t \rightarrow \infty$ and has nonpositive limit Lyapunov exponents (see, e.g. [71]).
} 
the strange attractor. Thus, the numerical computation of trajectory for a longer time may not lead to a more precise approximation of the Lyapunov exponents and dimension (see, also example (24) below). Also any long-time computation may be insufficient to reveal the limit values of LEs if the trajectory belongs to a transient chaotic set, which can be (almost) indistinguishable numerically from sustained chaos (see Figs. 9a, 10a). Note that there is no rigorous justification of the choice of $t$ and it is known that unexpected jumps of $\operatorname{dim}_{\mathrm{L}}(t, K)$ can occur (see, e.g. Fig. 7). Thus, for a finite time interval $t \in[0, T)$, it is reasonable to compute $\inf _{t \in[0, T)} \operatorname{dim}_{\mathrm{L}}(t, K)$ instead of $\operatorname{dim}_{\mathrm{L}}(T, K)$, but, at the same time, for any $T$ the value $\operatorname{dim}_{\mathrm{L}}(T, K)$ gives an upper estimate of $\operatorname{dim}_{\mathrm{H}} K$.

Finally, in the numerical experiments, based on the finite-time Lyapunov dimension definition (15) [19] and the Douady-Oesterlé theorem [79], we have

$$
\begin{aligned}
\operatorname{dim}_{\mathrm{H}} K & \leq \operatorname{dim}_{\mathrm{L}} K \approx \inf _{t \in[0, T]} \max _{u \in K_{\text {grid }}^{\varepsilon}} \operatorname{dim}_{\mathrm{L}}(t, u) \\
& =\inf _{t \in[0, T]} \max _{u \in K_{\text {grid }}^{\varepsilon}}\left(j(t, u)+\frac{\mathrm{LE}_{1}(t, u)+\cdots+\mathrm{LE}_{j(t, u)}(t, u)}{\left|\mathrm{LE}_{j(t, u)+1}(t, u)\right|}\right) \\
& \leq \max _{u \in K_{\text {grid }}^{\varepsilon}} \operatorname{dim}_{\mathrm{L}}(T, u) \approx \operatorname{dim}_{\mathrm{L}}(T, K) .
\end{aligned}
$$

Additionally, we can consider a set of random points in $K^{\varepsilon}$. If the maximums of the finite-time local Lyapunov dimensions for random points and grid points differ more than by $\delta$, then we decrease the distance between grid points. This may help to improve the reliability of the result and at the same time to ensure its repeatability.

Nowadays, there are several widely used approaches to numerical computation of the Lyapunov exponents; thus, it is important to state clearly how the LEs are being computed [92, p. 121]. Next, we demonstrate some differences in the approaches. For a certain $u_{0} \in \mathbb{R}^{3}$, to compute the finite-time Lyapunov exponents according to (16), one has to find the fundamental matrix $\Phi\left(T, u_{0}\right)=D \varphi^{T}\left(u_{0}\right)$ of (11) from the following variational equation:

$$
\left\{\begin{array}{l}
\dot{u}\left(t, u_{0}\right)=f\left(u\left(t, u_{0}\right)\right), \quad u\left(0, u_{0}\right)=u_{0}, \\
\dot{\Phi}\left(t, u_{0}\right)=J\left(u\left(t, u_{0}\right)\right) \Phi\left(t, u_{0}\right), \Phi\left(0, u_{0}\right)=I,
\end{array} \quad t \in[0, T],\right.
$$

and its singular value decomposition (SVD) ${ }^{6}$ :

$\Phi\left(T, u_{0}\right) \stackrel{\text { SVD }}{=} U\left(T, u_{0}\right) \Sigma\left(T, u_{0}\right) \mathrm{V}\left(T, u_{0}\right)^{*}$,

where $U\left(T, u_{0}\right)^{*} U\left(T, u_{0}\right) \equiv I \equiv \mathrm{V}\left(T, u_{0}\right)^{*} \mathrm{~V}\left(T, u_{0}\right)$, $\Sigma\left(T, u_{0}\right)=\operatorname{diag}\left\{\sigma_{1}\left(T, u_{0}\right), \sigma_{2}\left(T, u_{0}\right), \sigma_{3}\left(T, u_{0}\right)\right\}$ is a diagonal matrix composed by the singular values of $\Phi\left(T, u_{0}\right)$, and compute the finite-time Lyapunov exponents $\left\{\operatorname{LE}_{i}\left(T, u_{0}\right)\right\}_{1}^{3}$ from $\Sigma\left(T, u_{0}\right)$ as in (16). Further, we also need the QR decomposition ${ }^{7}$

$\Phi\left(T, u_{0}\right) \stackrel{\mathrm{QR}}{=} Q\left(T, u_{0}\right) R\left(T, u_{0}\right)$,

where $R\left(T, u_{0}\right)$ is an upper triangular matrix with nonnegative diagonal elements $\left\{R[i, i]=R[i, i]\left(T, u_{0}\right)\right\}_{1}^{3}$ and $Q\left(T, u_{0}\right)^{*} Q\left(T, u_{0}\right) \equiv I$.

To avoid the exponential growth of values in the computation, the time interval has to be represented as a union of sufficiently small intervals, for example, $(0, T]=(0, \tau] \cup(\tau, 2 \tau] \cdots \cup((k-1) \tau, k \tau=T]$. Then, using the cocycle property, the fundamental matrix can be represented as

$\Phi\left(k \tau, u_{0}\right)=\Phi\left(\tau, u_{k-1}\right) \ldots \Phi\left(\tau, u_{1}\right) \Phi\left(\tau, u_{0}\right)$.

Here, if $\Phi\left(m \tau, u_{0}\right)$ and $u_{m}=u\left(m \tau, u_{0}\right)$ are known, then $\Phi\left((m+1) \tau, u_{0}\right)=\Phi\left(\tau, u_{m}\right) \Phi\left(m \tau, u_{0}\right)$, where $\Phi\left(\tau, u_{m}\right)$ is the solution of initial value problem (19) with $u(0)=u_{m}$ on the time interval $[0, \tau]$.

By sequential QR decomposition of the product of matrices in (20), we get

$$
\begin{aligned}
\Phi & \left(k \tau, u_{0}\right)=\Phi\left(\tau, u_{k-1}\right) \ldots \Phi\left(\tau, u_{1}\right) \Phi\left(\tau, u_{0}\right) \\
& =\Phi\left(\tau, u_{k-1}\right) \ldots \overbrace{\Phi\left(\tau, u_{1}\right) Q_{1}^{0}} R_{1}^{0} \\
& =\ldots \stackrel{\mathrm{QR}}{=} \overbrace{Q_{k}^{0}}^{Q} \overbrace{R_{k}^{0} \ldots R_{1}^{0}}^{R} .
\end{aligned}
$$

Then, the matrix with singular values

$\Sigma\left(k \tau, u_{0}\right)=U^{*}\left(k \tau, u_{0}\right) \Phi\left(k \tau, u_{0}\right) V\left(k \tau, u_{0}\right)$

\footnotetext{
${ }^{6}$ See, for example, implementation in MATLAB or GNU Octave (https://octave-online.net): $[U, S, V]=\operatorname{svd}(A)$.

7 For example, it can be done by the Gram-Schmidt orthogonalization procedure or the Householder transformation. MATLAB and GNU Octave (https://octave-online.net) provide an implementation of the $\mathrm{QR}$ decomposition $[\mathrm{Q}, \mathrm{R}]=\mathrm{qr}(\mathrm{A})$. To have matrix $R$ with positive diagonal elements, one can additionally use $\mathrm{Q}=\mathrm{Q}^{*} \operatorname{diag}(\operatorname{sign}(\operatorname{diag}(\mathrm{R}))) ; \mathrm{R}=$ $R * \operatorname{diag}(\operatorname{sign}(\operatorname{diag}(R)))$. See also [106].
} 
in the SVD can be approximated by sequential $\mathrm{QR}$ decomposition of the product of matrices:

$$
\begin{aligned}
& \Sigma^{0}=\Phi\left(k \tau, u_{0}\right)^{*} Q_{k}^{0}=\left(R_{1}^{0}\right)^{*} \ldots\left(R_{k}^{0}\right)^{*} \stackrel{\mathrm{QR}}{=} Q_{k}^{1} R_{k}^{1} \ldots R_{1}^{1}, \\
& \Sigma^{1}=\left(Q_{k}^{0}\right)^{*} \Phi\left(k \tau, u_{0}\right) Q_{k}^{1}=\left(R_{1}^{1}\right)^{*} \ldots\left(R_{k}^{1}\right)^{*} \stackrel{\mathrm{QR}}{=} Q_{k}^{2} R_{k}^{2} \ldots R_{1}^{2}, \\
& \ldots
\end{aligned}
$$

where

$$
\Sigma^{p}=\left(R_{1}^{p}\right)^{*} . .\left(R_{k}^{p}\right)^{*}=\left(\begin{array}{ccc}
\sigma_{1}^{p} & 0 & 0 \\
\cdot & \sigma_{2}^{p} & 0 \\
\cdot & \cdot & \sigma_{3}^{p}
\end{array}\right)
$$

is a lower triangular matrix and $[107,108]$

$\sigma_{i}^{p}=R_{1}^{p}[i, i] \ldots R_{k}^{p}[i, i] \underset{p \rightarrow \infty}{\longrightarrow} \sigma_{i}\left(k \tau, u_{0}\right), \quad i=1,2,3$.

Thus, the finite-time Lyapunov exponents can be approximated as

$\operatorname{LE}_{i}\left(T, u_{0}\right) \approx \mathrm{LE}_{i}^{p}\left(k \tau, u_{0}\right)=\frac{1}{t} \ln \sigma_{i}^{p}=\frac{1}{k \tau} \sum_{l=1}^{k} \ln R_{l}^{p}[i, i]$.

The MATLAB implementation of the above method for the computation of finite-time Lyapunov exponents with the fixed number of iterations $p$ can be found, for example, in [9]. For large $k$, the convergence can be very rapid: for example, for the Lorenz system with the classical parameters $(r=28, \sigma=10, b=8 / 3, a=$ $0), k=1000$ and $\tau=1$, the number of approximations $p=1$ is taken in [108, p. 44]. For a more precise approximation of the finite-time Lyapunov exponents, we can adaptively choose $p=p(l), l=1, \ldots, k$ so as to obtain a uniform estimate:

$$
\max _{i=1,2,3}\left|\operatorname{LE}_{i}^{p(l)-1}\left(l \tau, u_{0}\right)-\operatorname{LE}_{i}^{p(l)}\left(l \tau, u_{0}\right)\right|<\delta . \quad l=1, \ldots, k .
$$

Remark that there is another widely used definition of the "Lyapunov exponents" via the exponential growth rates of norms of the fundamental matrix columns $\left(v_{1}\left(t, u_{0}\right), v_{2}\left(t, u_{0}\right), v_{3}\left(t, u_{0}\right)\right)=\Phi\left(t, u_{0}\right)$ : the finite-time Lyapunov characteristic exponents $\left\{\operatorname{LCE}_{i}\left(t, u_{0}\right)\right\}_{1}^{3}$ are the set $\left\{\frac{1}{t} \ln \left\|v^{i}\left(t, u_{0}\right)\right\|\right\}_{1}^{3}$ ordered by decreasing. ${ }^{8}$ Relying on ergodicity [84], Benettin et al. [109] (Benettin's algorithm; see also Wolf et al. [110]) approximate the LCEs by (21) with $p=0$ :

\footnotetext{
8 To obtain all possible limit values of the finite-time Lyapunov characteristic exponents (LCEs) [2] $\left\{\lim \sup _{t \rightarrow+\infty} \operatorname{LCE}_{i}\left(t, u_{0}\right)\right\}$ of linear system, one has to consider a normal fundamental matrix, whose sum of LCEs is less or equal to the sum of LCEs of any other fundamental matrix.
}

Table 1 Approximation of the finite-time Lyapunov exponents

\begin{tabular}{llll}
\hline$p$ & $\mathrm{LE}_{1,2}^{p}(5)$ & $\mathrm{LE}_{1,2}^{p}(25)$ & $\mathrm{LE}_{1,2}^{p}(100)$ \\
\hline 0 & 0 & 0 & 0 \\
1 & \pm 0.00797875 & \pm 0.04394912 & \pm 0.09360078 \\
2 & \pm 0.01585661 & \pm 0.07379280 & \pm 0.09986978 \\
3 & \pm 0.02353772 & \pm 0.08902280 & \pm 0.09999751 \\
4 & \pm 0.03093577 & \pm 0.09563887 & \pm 0.09999995 \\
5 & \pm 0.03797757 & \pm 0.09830568 & \pm 0.09999999 \\
10 & \pm 0.06638388 & \pm 0.09998593 & \pm 0.10000000 \\
50 & \pm 0.09993286 & \pm 0.09999999 & \pm 0.10000000 \\
100 & \pm 0.09999998 & \pm 0.09999999 & \pm 0.10000000 \\
\hline
\end{tabular}

$\operatorname{LCE}_{i}\left(k \tau, u_{0}\right) \approx \operatorname{LE}_{i}^{0}\left(k \tau, u_{0}\right)=\frac{1}{k \tau} \sum_{l=1}^{k} \ln R_{l}^{0}[i, i]$.

The LCEs may differ from LEs, thus, the corresponding Kaplan-Yorke formulas with respect to LEs and LCEs: $\operatorname{dim}_{\mathrm{L}}\left(t, u_{0}\right)=d_{\mathrm{L}}^{\mathrm{KY}}\left(\left\{\mathrm{LE}_{i}\left(t, u_{0}\right)\right\}_{1}^{3}\right)$ and $d_{\mathrm{L}}^{\mathrm{KY}}\left(\left\{\mathrm{LCE}_{i}\left(t, u_{0}\right)\right\}_{1}^{3}\right),{ }^{9}$ may not coincide. The following artificial analytical example demonstrates the possible difference between LEs and LCEs. The matrix $[19,86]$

$R(t)=\left(\begin{array}{cc}1 & g(t)-g^{-1}(t) \\ 0 & 1\end{array}\right), g(t)=\exp \left(\frac{t}{10}\right)$

has the following exact limit values

$$
\begin{aligned}
& \mathrm{LCE}_{1}=\lim _{t \rightarrow+\infty} t^{-1} \ln g(t)=0.1, \quad \mathrm{LCE}_{2}=0, \\
& \mathrm{LE}_{1,2}=\lim _{t \rightarrow+\infty} t^{-1} \ln g^{ \pm 1}(t)= \pm 0.1,
\end{aligned}
$$

where $\mathrm{LCE}_{2} \neq \mathrm{LE}_{2}$. For the finite-time values we have

$$
\begin{aligned}
& \operatorname{LCE}_{1}(t)=\frac{1}{t} \ln \left(\left(g(t)-\frac{1}{g(t)}\right)^{2}+1\right)^{\frac{1}{2}} \in(0,0.1], \\
& \operatorname{LCE}_{2}(t) \equiv 0, \\
& \operatorname{LE}_{1,2}(t) \equiv \mathrm{LE}_{1,2}= \pm 0.1 .
\end{aligned}
$$

Approximations by the above algorithm with $k=1$ are given in Table 1.

Remark that here the approximation of LCEs and LEs by Benettin's algorithm, i.e. by (23), becomes worse with increasing time:

\footnotetext{
9 Rabinovich et al. [81, p. 203, p. 262] refer this value as local dimension and note that it is a function of time and may be different in different parts of the attractor.
} 


$$
\begin{aligned}
& \operatorname{LCE}_{1}(t) \underset{t \rightarrow+0}{\longrightarrow} 0 \equiv \operatorname{LE}_{1}^{0}(t)=\frac{1}{t} \ln 1 \equiv 0, \\
& \operatorname{LCE}_{1}(t) \underset{t \rightarrow+\infty}{\longrightarrow} 0.1 \equiv \operatorname{LE}_{1}(t) \neq \operatorname{LE}_{1}^{0}(t)=\frac{1}{t} \ln 1 \equiv 0 .
\end{aligned}
$$

Thus, although the notions of LCEs and LEs often do not differ (see, e.g. Eckmann and Ruelle [15, p. 620, p. 650], Wolf et al. [110, p. 286, pp. 290-291] and Abarbanel et al. [17, p. 1363, p. 1364]) relying on the Oseledec ergodic theorem [84], in general case, the computations of LCEs by (21) and LEs by (23) may give unreliable results. See also numerical examples below and counterexamples in [111, p. 289], [97, p. 1083].

\subsection{Estimation of the Lyapunov dimension without integration of the system and the exact Lyapunov dimension}

While analytical computation of the Lyapunov exponents and Lyapunov dimension is impossible in a general case, they can be estimated by the eigenvalues of the symmetrized Jacobian matrix $[79,112]$. Let $\left\{\lambda_{i}(u)\right\}_{i=1}^{3}$ be the eigenvalues of the symmetrized Jacobian matrix $\frac{1}{2}\left(J(u)+J(u)^{*}\right)$, ordered so that $\lambda_{1}(u) \geq \lambda_{2}(u) \geq \lambda_{3}(u)$. The Kaplan-Yorke formula with respect to the ordered set of eigenvalues of the symmetrized Jacobian matrix [19] gives an upper estimation of the Lyapunov dimension:

$\operatorname{dim}_{\mathrm{L}} K \leq \sup _{u \in K} d_{\mathrm{L}}^{\mathrm{KY}}\left(\left\{\lambda_{i}(u)\right\}_{i=1}^{3}\right)$.

In a general case, one cannot get the same values of $\left\{\lambda_{i}(u)\right\}_{i=1}^{3}$ at different points $u$; thus, the maximum of $d_{\mathrm{L}}^{\mathrm{KY}}\left(\left\{\lambda_{i}(u)\right\}_{i}^{3}\right)$ on $K$ has to be computed. To avoid numerical localization of the set $K$, we can consider an analytical localization, e.g. by the absorbing set $\mathcal{B} \supset$ $K$. Then for the corresponding grid of points $\mathcal{B}_{\text {grid }}$, we expect in numerical experiments the following:

$$
\begin{aligned}
& \operatorname{dim}_{\mathrm{H}} K \leq \operatorname{dim}_{\mathrm{L}} K \leq \sup _{u \in K} d_{\mathrm{L}}^{\mathrm{KY}}\left(\left\{\lambda_{i}(u)\right\}_{1}^{3}\right) \\
& \leq \sup _{u \in \mathcal{B}} d_{\mathrm{L}}^{\mathrm{KY}}\left(\left\{\lambda_{i}(u)\right\}_{1}^{3}\right) \approx \max _{u \in \mathcal{B}_{\text {grid }}}(j(u) \\
& \left.\quad+\frac{\lambda_{1}(u)+\cdot \cdot+\lambda_{j(u)}(u)}{\left|\lambda_{j(u)+1}(u)\right|}\right) .
\end{aligned}
$$

If the Jacobian matrix $J\left(u_{\text {eq }}\right)$ at one of the equilibria has simple real eigenvalues: $\left\{\lambda_{i}\left(u_{\mathrm{eq}}\right)\right\}_{i=1}^{3}, \lambda_{i}\left(u_{\mathrm{eq}}\right) \geq$ $\lambda_{i+1}\left(u_{\mathrm{eq}}\right)$, then $[19,86]$ the invariance of the Lyapunov dimension with respect to linear change of variables implies

$\operatorname{dim}_{\mathrm{L}} u_{\mathrm{eq}}=d_{\mathrm{L}}^{\mathrm{KY}}\left(\left\{\lambda_{i}\left(u_{\mathrm{eq}}\right)\right\}_{i=1}^{3}\right)$.

If the maximum of local Lyapunov dimensions on the global attractors, which involves all equilibria, is achieved at an equilibrium point: $\operatorname{dim}_{\mathrm{L}} u_{\text {eq }}^{c r}=$ $\max _{u \in K} \operatorname{dim}_{\mathrm{L}} u$, then this allows one to get analytical formula for the exact Lyapunov dimension. ${ }^{10}$ In general, a conjecture on the Lyapunov dimension of selfexcited attractor $[19,114]$ is that for a typical system, the Lyapunov dimension of a self-excited attractor does not exceed the Lyapunov dimension of one of the unstable equilibria, the unstable manifold of which intersects with the basin of attraction and visualizes the attractor.

To avoid numerical computation of the eigenvalues, one can use an effective analytical approach $[19,22,115]$, which is based on a combination of the Douady-Oesterlé approach with the direct Lyapunov method: for example, in [22] for system (4) with $b=1$, it analytically obtained the following estimate

$\operatorname{dim}_{\mathrm{L}} K \leq 3-\frac{2(\sigma+2)}{\sigma+1+\sqrt{(\sigma-1)^{2}+\frac{16 r}{3 \sigma}}}$.

The proof of the above conjecture and analytical derivation of the exact Lyapunov dimension formula for system (4) with all possible parameters is an open problem.

In [116,117], it is demonstrated how the above technique can be effectively used to derive constructive upper bounds of the topological entropy of dynamical systems.

\section{The finite-time Lyapunov dimension in the case of hidden attractor and multistability}

Consider the dynamical system $\left\{\varphi^{t}\right\}_{t \geq 0}$, generated by system (4) with parameters (5), and its attractor $K$. Here, $\varphi^{t}\left(\left(x_{0}, y_{0}, z_{0}\right)\right)$ is a solution of (4) with the initial data $\left(x_{0}, y_{0}, z_{0}\right)$. Since the dynamical system $\left\{\varphi_{\mathrm{R}}^{t}\right\}_{t \geq 0}$, generated by the Rabinovich system (1), can be obtained from $\left\{\varphi^{t}\right\}_{t \geq 0}$ by the smooth transformation $\chi^{-1}$, inverse to (2), and inverse rescaling time (3) $t \rightarrow v_{1} t$, we have $\operatorname{dim}_{\mathrm{L}}\left(\left\{\varphi^{t}\right\}_{t \geq 0}, K\right)=$ $\operatorname{dim}_{\mathrm{L}}\left(\left\{\varphi_{\mathrm{R}}^{t}\right\}_{t \geq 0}, \chi^{-1}(K)\right)$. In our experiments, we consider system (4) with parameters $r=100, a=$

10 This term was suggested by Doering et al. in [113]. 


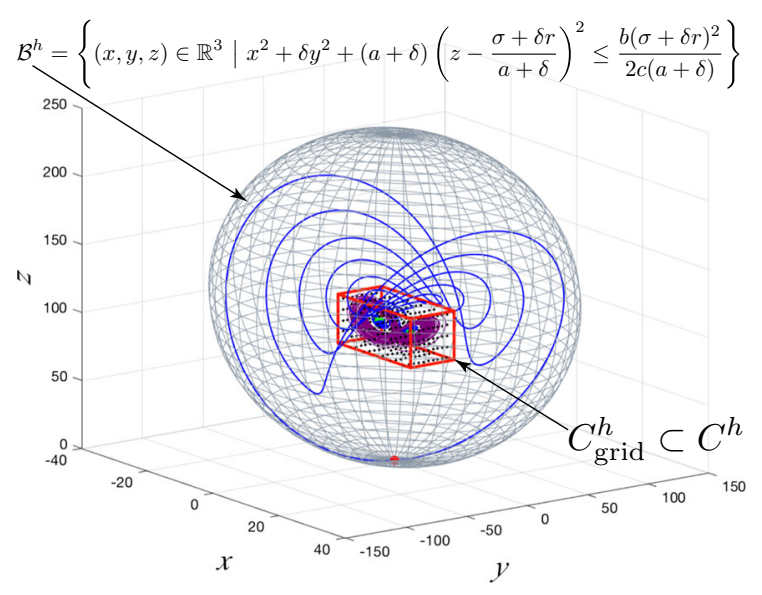

Fig. 6 Localization of the hidden attractor of system (4) with $r=$ $100, a=-9.965 \times 10^{-3}, b=7.7454 \times 10^{-2}$ by the absorbing set $\mathcal{B}^{h}$ with $\delta=-a+0.1$ and cuboid $C^{h}=[-11,11] \times$ $[-17,19] \times[80,117]$

$-9.965 \times 10^{-3}, b=7.7454 \times 10^{-2}$ corresponding to the hidden chaotic attractor.

In Fig. 6 is shown the grid of points $C_{\text {grid }}^{h}$ filling the hidden attractor: the grid of points fills cuboid $C^{h}=$ $[-11,11] \times[-17,19] \times[80,117]$ with the distance between points being equal to 0.5 . The time interval is [0, $T=100], k=1000, \tau=0.1$, and the integration method is MATLAB ode45 with predefined parameters. The infimum on the time interval is computed at the points $\left\{t_{i}\right\}_{1}^{k}$ with time step $\tau=t_{i+1}-t_{i}=0.1$. Note that if for a certain time $t=t_{i}$ the computed trajectory is out of the cuboid, the corresponding value of finite-time local Lyapunov dimension is not taken into account in the computation of maximum of the finitetime local Lyapunov dimension (there are trajectories with initial data in cuboid, which are attracted to the zero equilibria and belong to its stable manifold, e.g. system (4) with $x=y=0$ is $\dot{z}=-b z$ ). For the finitetime Lyapunov exponents (FTLEs) computation, we use MATLAB realization from [9] based on (21) with $p=2$. For computation of the finite-time Lyapunov characteristic exponents (FTLCEs), we use MATLAB realization from [118] based on (23).
For the considered set of parameters, we compute:

(i) Finite-time local Lyapunov dimensions $\operatorname{dim}_{\mathrm{L}}(100, \cdot)$ at the point $O_{1}=(0,1,98)$, which belongs to the grid $C_{\text {grid }}^{h}$, and at the point $O_{2}=$ $(0.0099,0.0995,0)$ on the unstable manifold of zero equilibrium $S_{0}$;

(ii) Maximum of the finite-time local Lyapunov dimensions at the points of grid, $\max _{u \in C_{\text {grid }}^{h}} \operatorname{dim}_{\mathrm{L}}(t, u)$, for the time points $t=t_{k}=0.1 k(k=$ $1, \ldots, 1000)$;

(iii) The corresponding values, given by the KaplanYorke formula with respect to finite-time Lyapunov characteristic exponents.

The results are given in Table 2. The dynamics of finite-time local Lyapunov dimensions for different points and their maximums on the grid of points are shown in Fig. 7.

For the absorbing set $\mathcal{B}^{h}$ and the corresponding grid of points $\mathcal{B}_{\text {grid }}^{h}$ (the distance between grid points is 5), by estimation (25), we get the following estimate:

$$
\begin{gathered}
\operatorname{dim}_{\mathrm{H}} K \leq \operatorname{dim}_{\mathrm{L}} K \leq \sup _{u \in \mathcal{B}^{h}} d_{\mathrm{L}}^{\mathrm{KY}}\left(\left\{\lambda_{j}(u)\right\}_{i=1}^{3}\right) \\
\approx \sup _{u \in \mathcal{B}_{\text {grid }}^{h}} d_{\mathrm{L}}^{\mathrm{KY}}\left(\left\{\lambda_{j}(u)\right\}_{i=1}^{3}\right)=2.97001 \ldots .
\end{gathered}
$$

Assuming $\sigma+1 \geq b$, the eigenvalues of the unstable zero equilibrium $S_{0}$ :

$$
\begin{aligned}
\lambda_{1,3}\left(S_{0}\right) & =-\frac{1}{2}\left[(\sigma+1) \mp \sqrt{(\sigma-1)^{2}+4 \sigma r}\right], \\
\lambda_{2}\left(S_{0}\right) & =-b,
\end{aligned}
$$

are ordered by decreasing, i.e. $\lambda_{1}\left(S_{0}\right)>\lambda_{2}\left(S_{0}\right) \geq$ $\lambda_{3}\left(S_{0}\right)$. Therefore, for the considered values of parameters by (26), we get

$$
\begin{aligned}
\operatorname{dim}_{\mathrm{L}} S_{0} & =d_{\mathrm{L}}^{\mathrm{KY}}\left(\left\{\lambda_{i}\left(S_{0}\right)\right\}_{i=1}^{3}\right)=2+\frac{\lambda_{1}\left(S_{0}\right)+\lambda_{2}\left(S_{0}\right)}{\left|\lambda_{3}\left(S_{0}\right)\right|} \\
& =3-\frac{2(\sigma+b+1)}{(\sigma+1)+\sqrt{(\sigma-1)^{2}+4 \sigma r}}=2.8111 \ldots .
\end{aligned}
$$

The above numerical experiments lead us to the following important remarks. While the Lyapunov dimen-

Table 2 Numerical estimation of finite-time Lyapunov dimension in the case of hidden attractor (see Fig. 3)

\begin{tabular}{lllll}
\hline & $\begin{array}{l}t=100 \\
u=(0,1,98)\end{array}$ & $\begin{array}{l}t=100 \\
u=(0.0099,0.0995,0)\end{array}$ & $\begin{array}{c}t=100 \\
\max _{u \in C_{\text {grid }}^{h}}\end{array}$ & $\begin{array}{c}\inf \max \\
t \in[0,100]\end{array}$ \\
\hline $\operatorname{dim}_{\mathrm{L}}(t, u)=d_{\mathrm{L}}^{\mathrm{KY}}\left(\left\{\mathrm{LE}_{i}(t, u)\right\}_{i=1}^{3}\right)$ & 2.1474 & 1.4987 & 2.2063 & 2.2050 \\
$d_{\mathrm{L}}^{\mathrm{KY}}\left(\left\{\mathrm{LCE}_{i}(t, u)\right\}_{i=1}^{3}\right)$ & 2.1338 & 1.1213 & 2.2105 & 2.2076 \\
\hline
\end{tabular}




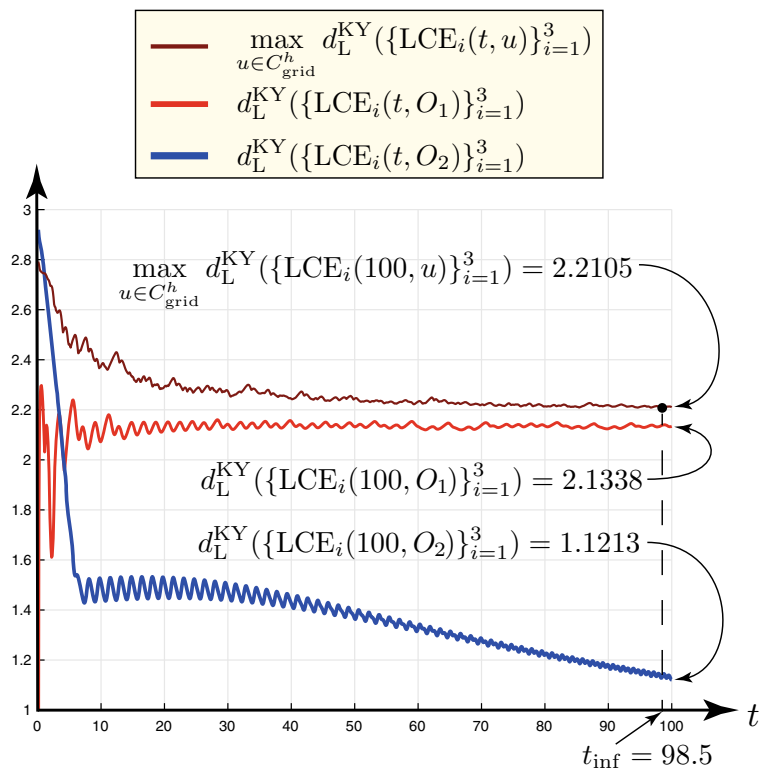

Fig. 7 Dynamics of the finite-time local Lyapunov dimension estimations on the time interval $t \in[0,100]$ : the maximum on the grid of points (dark red), at the point $O_{1}=(0,1,98) \in C_{\text {grid }}^{h}$

sion, unlike the Hausdorff dimension, is not a dimension in the rigorous sense [119] (e.g. the Lyapunov dimension of the saddle point $S_{0}$ in (28) is noninteger), it gives an upper estimate of the Hausdorff dimension. If the attractor $K$ or the corresponding absorbing set $\mathcal{B} \supset K$ is known [see, e.g. (8)] and our purpose is to demonstrate that $\operatorname{dim}_{\mathrm{H}} K \leq \operatorname{dim}_{\mathrm{L}} K<3$, then it can be achieved without integration of the considered dynamical system [see, e.g. (27)]. If our purpose is to get a precise estimation of the Hausdorff dimension, then we can use (18) and compute the finite-time Lyapunov dimension, i.e. to find the maximum of the finitetime local Lyapunov dimensions on a grid of points for a certain time. To be able to repeat a computation of finite-time Lyapunov dimension, one need to know the initial points of considered trajectories $\left\{u_{i}\right\}_{i=1}^{k}=K_{\text {grid }}$ on the set $K$, time interval $(0, T]=\bigcup_{i=0}^{k-1}\left(t_{i}, t_{i+1}\right]$ and how the finite-time Lyapunov exponents were computed (e.g. by (23), (21), or (22) with the parameter $\delta)$.

\section{Computation of the Lyapunov dimension and transient chaos}

Consider an example, which demonstrates difficulties in the reliable numerical computation of the Lyapunov

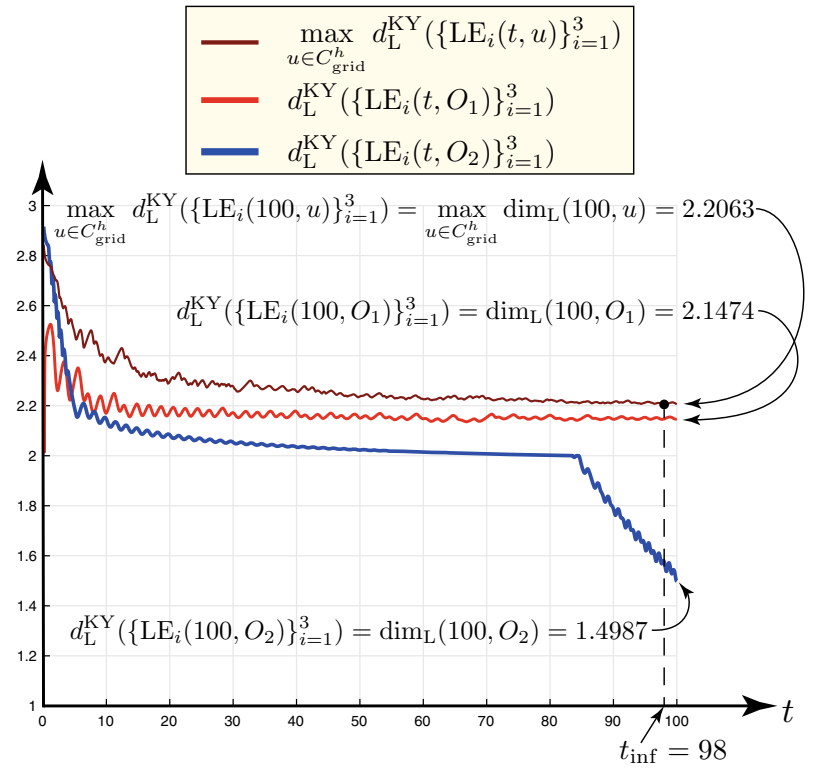

(light red), at the point $O_{2}=(0.0099,0.0995,0)$ from the onedimensional unstable manifold of $S_{0}$ (blue). (Color figure online)

dimension (i.e. numerical approximation of the limit values of the finite-time Lyapunov dimensions).

Consider system (4) with parameters $r=6.485$, $a=-0.5, b=0.85$ for which equilibrium $S_{0}$ is a saddle point and equilibria $S_{ \pm}$are stable focus-nodes and integrate numerically ${ }^{11}$ the trajectory with initial data $u_{0}=(0.5,0.5,0.5)$ in the vicinity of the $S_{0}$. We discard the part of the trajectory, corresponding to the initial transition process (for $\left[0, t_{\mathrm{tp}}=25,000\right]$ ), and get the point $u_{\text {init }}=(-2.089862710574761$, $-2.500837780529156,2.776106323157132)$. Further, we numerically approximate the finite-time Lyapunov exponents and dimension for the time interval $[0, T]$ by (23) (see MATLAB code in [120]).

11 Our experiment was carried out on the $2.5 \mathrm{GHz}$ Intel Core i7 MacBook Pro laptop. For numerical integration, we use MATLAB R2016b. To simplify the repetition of results, we use the single-step fifth-order Runge-Kutta method ode 5 from https://www.mathworks.com/matlabcentral/answers/98293-isthere-a-fixed-step-ordinary-differential-equation-ode-solver-inmatlab-8-0-r2012b\#answer107643. Corresponding numerical simulation of the considered trajectory can be performed using the following code: phiT_u = feval ('ode5', a $(t, u)[-3.2425 * u(1)+3.2425 * u(2)+0.5 *$ $u(2) * u(3) ; 6.485 * u(1)-u(2)-u(1) * u(3)$; $u(1) * u(2)-0.85 * u(3)], 0: 0.01: 15295$, $[-2.089862710574761,-2.500837780529156$, 2.776106323157132]); 


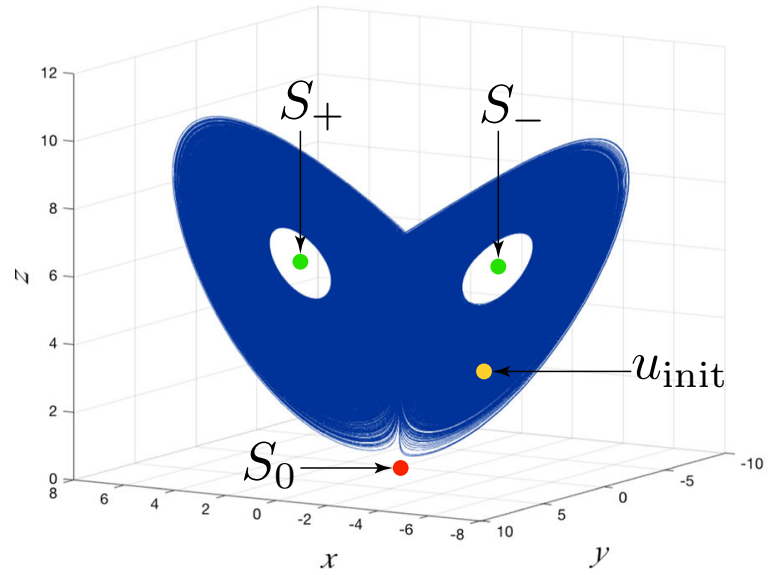

(a)

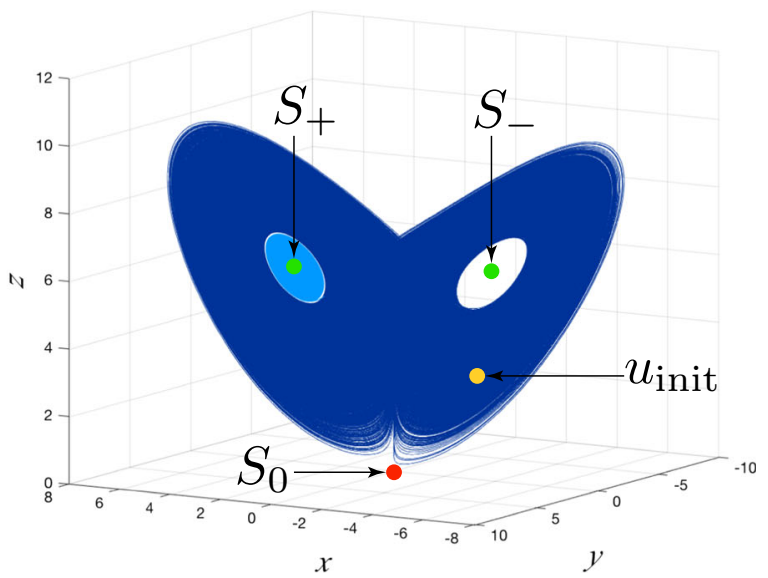

(b)

Fig. 8 The trajectory forms a chaotic set, which looks like an "attractor" (navy blue) and then tends to $S_{+}$(cyan). a Trajectory $u\left(t, u_{\text {init }}\right.$ ) for $t \in\left[0, T_{1}\right], T_{1} \approx 15,295$. b Trajectory $u\left(t, u_{\text {init }}\right)$ for $t \in[0, T], T=500,000$. (Color figure online)

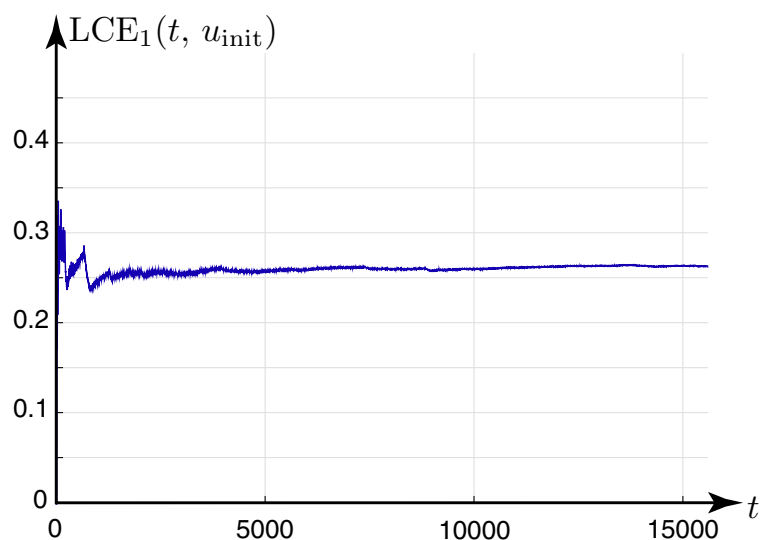

(a)

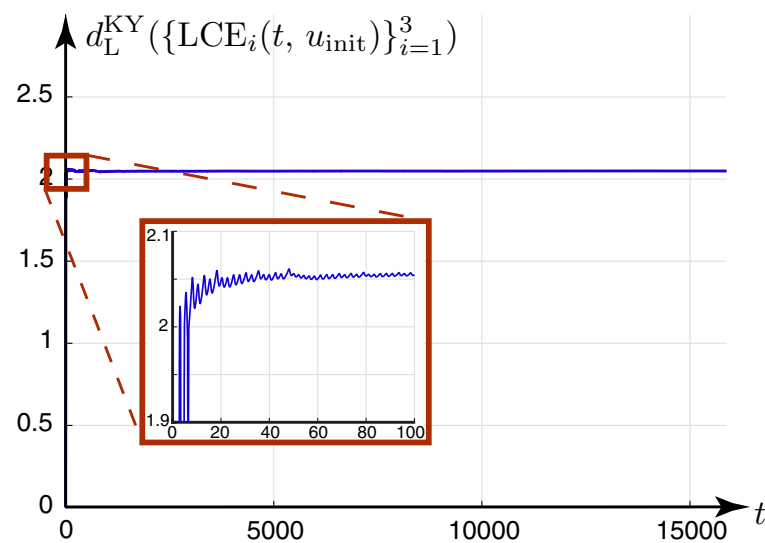

(b)

Fig. 9 Numerical computation of $\operatorname{LCE}_{1}\left(t, u_{\text {init }}\right)$ and $d_{\mathrm{L}}^{\mathrm{KY}}\left(\left\{\mathrm{LCE}_{i}\left(t, u_{\text {init }}\right)\right\}_{i=1}^{3}\right)$ for the time interval $\left[0, T_{1} \approx 15,295\right]$. a LCE $1\left(t, u_{\text {init }}\right)$, $t \in\left[0, T_{1}\right], T_{1} \approx 15,295 . \mathbf{b} d_{\mathrm{L}}^{\mathrm{KY}}\left(\left\{\operatorname{LCE}_{i}\left(t, u_{\mathrm{init}}\right)\right\}_{i=1}^{3}\right), t \in\left[0, T_{1}\right], T_{1} \approx 15,295$

The trajectory computed on the time interval $\left[0, T_{1} \approx 15295\right]$ traces a chaotic set in the phase space, which looks like an "attractor" (see Fig. 8a, Fig. 9a). Further integration with $t>T_{1}$ leads to the collapse of the "attractor", i.e. the "attractor" (see Fig. 8a) turns out to be a transient chaotic set (see Fig. 8b). However on the time interval $t \in\left[0, T_{3} \approx 431,560\right]$, we have $\operatorname{LCE}_{1}\left(t, u_{\text {init }}\right)>0$ (see Fig. 10a) and, thus, may conclude that the behaviour is chaotic, and for the time interval $t \in\left[0, T_{2} \approx 223,447\right]$, we have $d_{\mathrm{L}}^{\mathrm{KY}}\left(\left\{\mathrm{LCE}_{i}\left(t, u_{\text {init }}\right)\right\}_{i=1}^{3}\right)>2$ (see Fig. 10b). This effect is due to the fact that the finite-time Lyapunov exponents and finite-time Lyapunov dimension are the values averaged over the considered time interval.
Since the lifetime of transient chaotic process can be extremely long and taking into account the limitations of reliable integration of chaotic ODEs, even longtime numerical computation of the finite-time Lyapunov exponents and the finite-time Lyapunov dimension does not necessarily lead to a relevant approximation of the Lyapunov exponents and the Lyapunov dimension [see also effects in (24)].

\section{Conclusion}

In this work, the Rabinovich system, describing the process of interaction between waves in plasma, is 


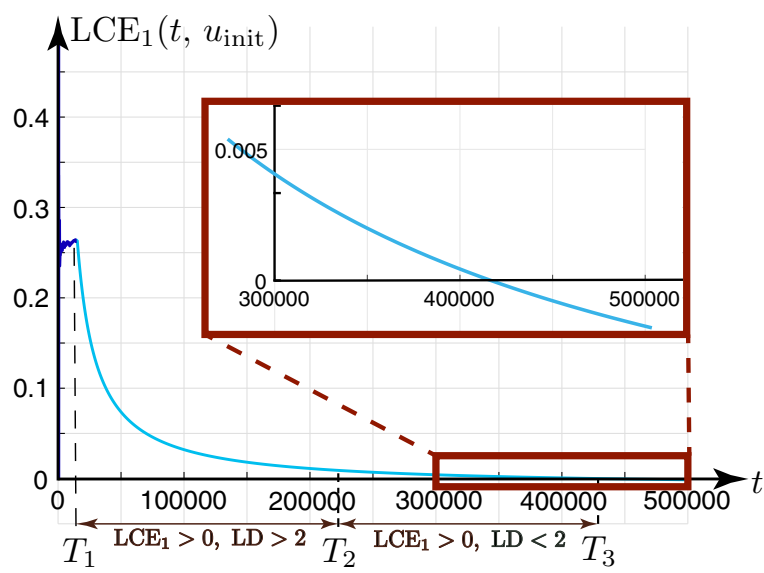

(a)

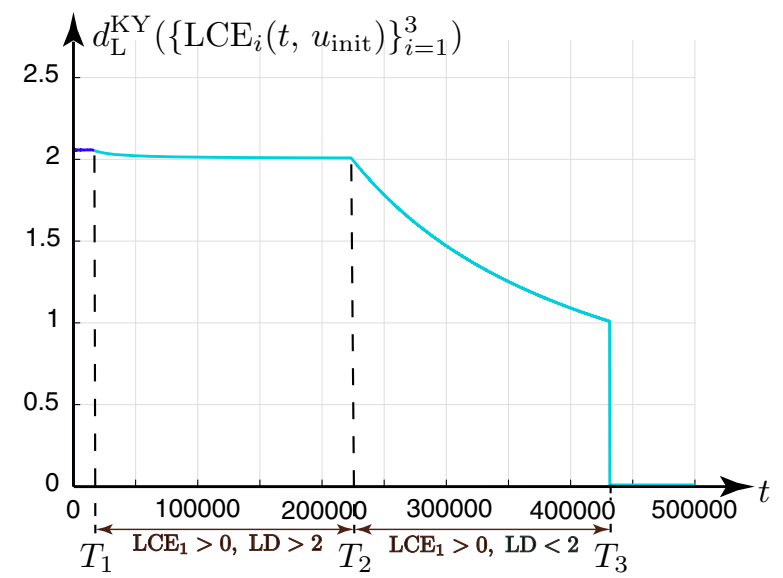

(b)

Fig. 10 Numerical computation of $\operatorname{LCE}_{1}\left(t, u_{\text {init }}\right)$ and $d_{\mathrm{L}}^{\mathrm{KY}}\left(\left\{\mathrm{LCE}_{i}\left(t, u_{\text {init }}\right)\right\}_{i=1}^{3}\right)$ for the time interval $\left[0,5 \cdot 10^{5}\right] . \mathbf{a} \mathrm{LCE}{ }_{1}\left(t, u_{\text {init }}\right)$, $t \in[0, T], T=5 \cdot 10^{5} . \mathbf{b} d_{\mathrm{L}}^{\mathrm{KY}}\left(\left\{\operatorname{LCE}_{i}\left(t, u_{\text {init }}\right)\right\}_{i=1}^{3}\right), t \in[0, T], T=5 \cdot 10^{5}$

considered. It is shown that the methods of numerical continuation and perpetual point are helpful for the localization and understanding of hidden attractor in the Rabinovich system. For the study of dimension of the hidden attractor, the concept of the finite-time Lyapunov dimension is developed. An approach to reliable numerical estimation of the finite-time Lyapunov exponents [see (21) and (22)] and finite-time Lyapunov dimension [see (18)] is suggested. Various numerical estimates of the finite-time Lyapunov dimension for the hidden attractor in the case of multistability are given.

Acknowledgements The work in Sects. 1-4 is done within the joint Grant from DST and RFBR (INT/RUS/RFBR/P-230 and 16-51-45002); Sects. 5-7 are done within Russian Science Foundation Project (14-21-00041).

Open Access This article is distributed under the terms of the Creative Commons Attribution 4.0 International License (http:// creativecommons.org/licenses/by/4.0/), which permits unrestricted use, distribution, and reproduction in any medium, provided you give appropriate credit to the original author(s) and the source, provide a link to the Creative Commons license, and indicate if changes were made.

\section{References}

1. Poincare, H.: Les methodes nouvelles de la mecanique celeste, vol. 1-3. Gauthiers-Villars, Paris (1892, 1893, 1899) (English transl. edited by D. Goroff: American Institute of Physics, NY, 1993)

2. Lyapunov, A.: The General Problem of the Stability of Motion, Kharkov (1892) (English transl.: Academic Press, NY, 1966). (in Russian)
3. Leonov, G., Reitmann, V.: Attraktoreingrenzung fur Nichtlineare Systeme. Teubner, Leipzig (1987) (in German)

4. Hilbert, D.: Mathematical problems. Bull. Am. Math. Soc. 8: 437-479 (1901-1902)

5. Leonov, G., Kuznetsov, N.: On differences and similarities in the analysis of Lorenz, Chen, and Lu systems. Appl. Math. Comput. 256, 334-343 (2015)

6. Pisarchik, A., Feudel, U.: Control of multistability. Phys. Rep. 540(4), 167-218 (2014)

7. Leonov, G., Kuznetsov, N.: Hidden attractors in dynamical systems. From hidden oscillations in Hilbert-Kolmogorov, Aizerman, and Kalman problems to hidden chaotic attractors in Chua circuits. Int. J. Bifurc Chaos 23(1), 1330002 (2013)

8. Kuznetsov, N., Leonov, G.: Hidden attractors in dynamical systems: systems with no equilibria, multistability and coexisting attractors. IFAC Proc. Vol. 47, 5445-5454 (2014)

9. Leonov, G., Kuznetsov, N., Mokaev, T.: Homoclinic orbits, and self-excited and hidden attractors in a Lorenz-like system describing convective fluid motion. Eur. Phys. J. Special Top. 224(8), 1421-1458 (2015)

10. Kuznetsov, N.: Hidden attractors in fundamental problems and engineering models. A short survey. Lecture Notes Electr. Eng. 371, 13-25 (2016) (Plenary lecture at International Conference on Advanced Engineering Theory and Applications 2015)

11. Grebogi, C., Ott, E., Yorke, J.: Fractal basin boundaries, long-lived chaotic transients, and unstable-unstable pair bifurcation. Phys. Rev. Lett. 50(13), 935 (1983)

12. Dudkowski, D., Jafari, S., Kapitaniak, T., Kuznetsov, N., Leonov, G., Prasad, A.: Hidden attractors in dynamical systems. Phys. Rep. 637, 1-50 (2016)

13. Kaplan, J., Yorke, J.: Chaotic behavior of multidimensional difference equations. In: Peitgen, H.-O., Walther, H.-O. (eds.) Functional Differential Equations and Approximations of Fixed Points, pp. 204-227. Springer, Berlin (1979) 
14. Grassberger, P., Procaccia, I.: Measuring the strangeness of strange attractors. Phys. D Nonlinear Phenom. 9(1-2), 189-208 (1983)

15. Eckmann, J.P., Ruelle, D.: Ergodic theory of chaos and strange attractors. Rev. Mod. Phys. 57(3), 617-656 (1985)

16. Constantin, P., Foias, C., Temam, R.: Attractors representing turbulent flows. Mem. Am. Math. Soc. 53(314), 1-67 (1985)

17. Abarbanel, H., Brown, R., Sidorowich, J., Tsimring, L.: The analysis of observed chaotic data in physical systems. Rev. Mod. Phys. 65(4), 1331-1392 (1993)

18. Boichenko, V., Leonov, G., Reitmann, V.: Dimension Theory for Ordinary Differential Equations. Teubner, Stuttgart (2005)

19. Kuznetsov, N.: The Lyapunov dimension and its estimation via the Leonov method. Phys. Lett. A 380(25-26), 21422149 (2016)

20. Rabinovich, M.I.: Stochastic self-oscillations and turbulence. Soviet Phys Uspekhi 21(5), 443-469 (1978)

21. Pikovski, A., Rabinovich, M., Trakhtengerts, V.: Onset of stochasticity in decay confinement of parametric instability. Sov. Phys. JETP 47, 715-719 (1978)

22. Leonov, G., Boichenko, V.: Lyapunov's direct method in the estimation of the Hausdorff dimension of attractors. Acta Appl. Math. 26(1), 1-60 (1992)

23. Lorenz, E.: Deterministic nonperiodic flow. J. Atmos. Sci. 20(2), 130-141 (1963)

24. Chueshov, I.: Introduction to the Theory of Infinitedimensional Dissipative Systems. Electronic Library of Mathematics. ACTA, Kharkiv (2002)

25. Leonov, G., Kuznetsov, N., Vagaitsev, V.: Localization of hidden Chua's attractors. Phys. Lett. A 375(23), 2230-2233 (2011)

26. Sommerfeld, A.: Beitrage zum dynamischen ausbau der festigkeitslehre. Z. Vereins Dtsch. Ing. 46, 391-394 (1902). (in German)

27. Kiseleva, M., Kuznetsov, N., Leonov, G.: Hidden attractors in electromechanical systems with and without equilibria. IFAC-PapersOnLine 49(14), 51-55 (2016)

28. Leonov, G., Kuznetsov, N.: Algorithms for searching for hidden oscillations in the Aizerman and Kalman problems. Dokl. Math. 84(1), 475-481 (2011)

29. Aizerman, M.A.: On a problem concerning the stability in the large of dynamical systems. Uspekhi Mat. Nauk 4, 187-188 (1949). (in Russian)

30. Kalman, R.E.: Physical and mathematical mechanisms of instability in nonlinear automatic control systems. Trans. ASME 79(3), 553-566 (1957)

31. Bautin, N.N.: On the number of limit cycles generated on varying the coefficients from a focus or centre type equilibrium state. Dokl. Akad. Nauk SSSR 24(7), 668-671 (1939). (in Russian)

32. Kuznetsov, N., Kuznetsova, O., Leonov, G.: Visualization of four normal size limit cycles in two-dimensional polynomial quadratic system. Differ. Equ. Dyn. Syst. 21(1-2), 29-34 (2013)

33. Kuznetsov, N., Leonov, G., Vagaitsev, V.: Analyticalnumerical method for attractor localization of generalized Chua's system. IFAC Proc. Vol. 43(11), 29-33 (2010)

34. Bragin, V., Vagaitsev, V., Kuznetsov, N., Leonov, G.: Algorithms for finding hidden oscillations in nonlinear systems.
The Aizerman and Kalman conjectures and Chua's circuits. J. Comput. Syst. Sci. Int. 50(4), 511-543 (2011)

35. Leonov, G., Kuznetsov, N., Vagaitsev, V.: Hidden attractor in smooth Chua systems. Phys. D Nonlinear Phenom. 241(18), 1482-1486 (2012)

36. Kuznetsov, N., Kuznetsova, O., Leonov, G., Vagaitsev, V.: Analytical-numerical localization of hidden attractor in electrical Chua's circuit. Lecture Notes Electr. Eng. 174(4), 149-158 (2013)

37. Kiseleva, M., Kudryashova, E., Kuznetsov, N., Kuznetsova, O., Leonov, G., Yuldashev, M., Yuldashev, R.: Hidden and self-excited attractors in Chua circuit: synchronization and SPICE simulation. Int. J. Parallel Emergent Distrib. Syst. (2017). https://doi.org/10.1080/17445760.2017.1334776

38. Stankevich, N., Kuznetsov, N., Leonov, G., Chua, L.: Scenario of the birth of hidden attractors in the Chua circuit. Int. J. Bifurc. Chaos 27(12), 1730038 (2017)

39. Burkin, I., Khien, N.: Analytical-numerical methods of finding hidden oscillations in multidimensional dynamical systems. Differ. Equ. 50(13), 1695-1717 (2014)

40. Li, C., Sprott, J.: Coexisting hidden attractors in a 4-D simplified Lorenz system. Int. J. Bifurc. Chaos 24(03), 1450034 (2014)

41. Li, Q., Zeng, H., Yang, X.S.: On hidden twin attractors and bifurcation in the Chua's circuit. Nonlinear Dyn. 77(1-2), 255-266 (2014)

42. Pham, V.T., Rahma, F., Frasca, M., Fortuna, L.: Dynamics and synchronization of a novel hyperchaotic system without equilibrium. Int. J. Bifurc. Chaos 24(06), 1450087 (2014)

43. Chen, M., Li, M., Yu, Q., Bao, B., Xu, Q., Wang, J.: Dynamics of self-excited attractors and hidden attractors in generalized memristor-based Chua's circuit. Nonlinear Dyn. 81, 215-226 (2015)

44. Kuznetsov, A., Kuznetsov, S., Mosekilde, E., Stankevich, N.: Co-existing hidden attractors in a radio-physical oscillator system. J. Phys. A Math. Theor. 48, 125101 (2015)

45. Saha, P., Saha, D., Ray, A., Chowdhury, A.: Memristive non-linear system and hidden attractor. Eur. Phys. J. Special Top. 224(8), 1563-1574 (2015)

46. Semenov, V., Korneev, I., Arinushkin, P., Strelkova, G., Vadivasova, T., Anishchenko, V.: Numerical and experimental studies of attractors in memristor-based Chua's oscillator with a line of equilibria. Noise-induced effects. Eur. Phys. J. Special Top. 224(8), 1553-1561 (2015)

47. Sharma, P., Shrimali, M., Prasad, A., Kuznetsov, N., Leonov, G.: Control of multistability in hidden attractors. Eur. Phys. J. Special Top. 224(8), 1485-1491 (2015)

48. Zhusubaliyev, Z., Mosekilde, E., Churilov, A., Medvedev, A.: Multistability and hidden attractors in an impulsive Goodwin oscillator with time delay. Eur. Phys. J. Special Top. 224(8), 1519-1539 (2015)

49. Wei, Z., Yu, P., Zhang, W., Yao, M.: Study of hidden attractors, multiple limit cycles from Hopf bifurcation and boundedness of motion in the generalized hyperchaotic Rabinovich system. Nonlinear Dyn. 82(1), 131-141 (2015)

50. Danca, M.F., Kuznetsov, N., Chen, G.: Unusual dynamics and hidden attractors of the Rabinovich-Fabrikant system. Nonlinear Dyn. 88, 791-805 (2017)

51. Jafari, S., Pham, V.T., Golpayegani, S., Moghtadaei, M., Kingni, S.: The relationship between chaotic maps and 
some chaotic systems with hidden attractors. Int. J. Bifurcat. Chaos 26(13), 1650211 (2016)

52. Menacer, T., Lozi, R., Chua, L.: Hidden bifurcations in the multispiral Chua attractor. Int. J. Bifurc. Chaos 26(14), 1630039 (2016)

53. Ojoniyi, O., Njah, A.: A 5D hyperchaotic Sprott B system with coexisting hidden attractors. Chaos Solitons Fractals 87, 172-181 (2016)

54. Pham, V.T., Volos, C., Jafari, S., Vaidyanathan, S., Kapitaniak, T., Wang, X.: A chaotic system with different families of hidden attractors. Int. J. Bifurc. Chaos 26(08), 1650139 (2016)

55. Rocha, R., Medrano-T, R.O.: Finding hidden oscillations in the operation of nonlinear electronic circuits. Electr. Lett. 52(12), 1010-1011 (2016)

56. Wei, Z., Pham, V.T., Kapitaniak, T., Wang, Z.: Bifurcation analysis and circuit realization for multiple-delayed Wang-Chen system with hidden chaotic attractors. Nonlinear Dyn. 85(3), 1635-1650 (2016)

57. Zelinka, I.: Evolutionary identification of hidden chaotic attractors. Eng. Appl. Artif. Intell. 50, 159-167 (2016)

58. Borah, M., Roy, B.: Hidden attractor dynamics of a novel non-equilibrium fractional-order chaotic system and its synchronisation control. In: 2017 Indian Control Conference (ICC), pp. 450-455 (2017)

59. Brzeski, P., Wojewoda, J., Kapitaniak, T., Kurths, J., Perlikowski, P.: Sample-based approach can outperform the classical dynamical analysis-experimental confirmation of the basin stability method. Sci. Rep. 7, 6121 (2017)

60. Feng, Y., Pan, W.: Hidden attractors without equilibrium and adaptive reduced-order function projective synchronization from hyperchaotic Rikitake system. Pramana 88(4), 62 (2017)

61. Jiang, H., Liu, Y., Wei, Z., Zhang, L.: Hidden chaotic attractors in a class of two-dimensional maps. Nonlinear Dyn. 85(4), 2719-2727 (2016)

62. Kuznetsov, N., Leonov, G., Yuldashev, M., Yuldashev, R.: Hidden attractors in dynamical models of phase-locked loop circuits: limitations of simulation in MATLAB and SPICE. Commun. Nonlinear Sci. Numer. Simul. 51, 3949 (2017)

63. Ma, J., Wu, F., Jin, W., Zhou, P., Hayat, T.: Calculation of Hamilton energy and control of dynamical systems with different types of attractors. Chaos Interdiscip. J. Nonlinear Sci. 27(5), 053108 (2017)

64. Messias, M., Reinol, A.: On the formation of hidden chaotic attractors and nested invariant tori in the Sprott A system. Nonlinear Dyn. 88(2), 807-821 (2017)

65. Singh, J., Roy, B.: Multistability and hidden chaotic attractors in a new simple 4-D chaotic system with chaotic 2-torus behaviour. Int. J. Dyn. Control (2017). https://doi.org/10. 1007/s40435-017-0332-8

66. Volos, C., Pham, V.T., Zambrano-Serrano, E., MunozPacheco, J.M., Vaidyanathan, S., Tlelo-Cuautle, E.: Analysis of a 4-D hyperchaotic fractional-order memristive system with hidden attractors. In: Vaidyanathan, S., Volos, C. (eds.) Advances in Memristors, Memristive Devices and Systems, pp. 207-235. Springer, Berlin (2017)

67. Wei, Z., Moroz, I., Sprott, J., Akgul, A., Zhang, W.: Hidden hyperchaos and electronic circuit application in a 5D self- exciting homopolar disc dynamo. Chaos 27(3), 033101 (2017)

68. Zhang, G., Wu, F., Wang, C., Ma, J.: Synchronization behaviors of coupled systems composed of hidden attractors. Int. J. Mod. Phys. B 31, 1750180 (2017)

69. Lai, Y., Tel, T.: Transient Chaos: Complex Dynamics on Finite Time Scales. Springer, New York (2011)

70. Danca, M.F., Kuznetsov, N.: Hidden chaotic sets in a Hopfield neural system. Chaos Solitons Fractals 103, 144-150 (2017)

71. Chen, G., Kuznetsov, N., Leonov, G., Mokaev, T.: Hidden attractors on one path: Glukhovsky-Dolzhansky, Lorenz, and Rabinovich systems. Int. J. Bifurc. Chaos 27(8), 1750115 (2017)

72. Kuznetsov, N., Leonov, G., Mokaev, T., Seledzhi, S.: Hidden attractor in the Rabinovich system, Chua circuits and PLL. AIP Conf. Proc. 1738(1), 210008 (2016)

73. Leonov, G., Kuznetsov, N., Mokaev, T.: Hidden attractor and homoclinic orbit in Lorenz-like system describing convective fluid motion in rotating cavity. Commun. Nonlinear Sci. Numer. Simul. 28, 166-174 (2015)

74. Prasad, A.: Existence of perpetual points in nonlinear dynamical systems and its applications. Int. J. Bifurc. Chaos 25(2), 1530005 (2015)

75. Dudkowski, D., Prasad, A., Kapitaniak, T.: Perpetual points and hidden attractors in dynamical systems. Phys. Lett. A 379(40-41), 2591-2596 (2015)

76. Prasad, A.: A note on topological conjugacy for perpetual points. Int. J. Nonlinear Sci. 21(1), 60-64 (2016)

77. Nazarimehr, F., Saedi, B., Jafari, S., Sprott, J.: Are perpetual points sufficient for locating hidden attractors? Int. J. Bifurc. Chaos 27(03), 1750037 (2017)

78. Dudkowski, D., Prasad, A., Kapitaniak, T.: Perpetual points: new tool for localization of coexisting attractors in dynamical systems. Int. J. Bifurc. Chaos 27(04), 1750063 (2017)

79. Douady, A., Oesterle, J.: Dimension de Hausdorff des attracteurs. C.R. Acad. Sci. Paris Ser. A (in French) 290(24), 1135-1138 (1980)

80. Hunt, B.: Maximum local Lyapunov dimension bounds the box dimension of chaotic attractors. Nonlinearity 9(4), 845-852 (1996)

81. Rabinovich, M., Ezersky, A., Weidman, P.: The Dynamics of Patterns. World Scientific, Singapore (2000)

82. Ruelle, D., Takens, F.: On the nature of turbulence. Commun. Math. Phys. 20(3), 167-192 (1971)

83. Grebogi, C., Ott, E., Yorke, J.: Chaos, strange attractors, and fractal basin boundaries in nonlinear dynamics. Science 238(4827), 632-638 (1987)

84. Oseledets, V.: A multiplicative ergodic theorem. Characteristic Lyapunov exponents of dynamical systems. Trudy Mosk. Matematicheskogo Obshchestva 19, 179-210 (1968). (in Russian)

85. Vallejo, J., Sanjuan, M.: Predictability of Chaotic Dynamics: A Finite-time Lyapunov Exponents Approach. Springer, Cham (2017)

86. Kuznetsov, N., Alexeeva, T., Leonov, G.: Invariance of Lyapunov exponents and Lyapunov dimension for regular and irregular linearizations. Nonlinear Dyn. 85(1), 195201 (2016) 
87. Ledrappier, F.: Some relations between dimension and Lyapunov exponents. Commun. Math. Phy. 81(2), 229-238 (1981)

88. Frederickson, P., Kaplan, J., Yorke, E., Yorke, J.: The Lyapunov dimension of strange attractors. J. Differ. Equ. 49(2), 185-207 (1983)

89. Farmer, J., Ott, E., Yorke, J.: The dimension of chaotic attractors. Phys. D Nonlinear Phenom. 7(1-3), 153-180 (1983)

90. Dellnitz, M., Junge, O.: Set oriented numerical methods for dynamical systems. In: Handbook of Dynamical Systems, vol. 2. Elsevier, Amsterdam, pp. 221-264 (2002)

91. Barreira, L., Schmeling, J.: Sets of "Non-typical" points have full topological entropy and full Hausdorff dimension. Israel J. Math. 116(1), 29-70 (2000)

92. Cvitanović, P., Artuso, R., Mainieri, R., Tanner, G., Vattay, G.: Chaos: Classical and Quantum. Niels Bohr Institute, Copenhagen. http://ChaosBook.org (2016)

93. Ott, W., Yorke, J.: When Lyapunov exponents fail to exist. Phys. Rev. E 78, 056203 (2008)

94. Young, L.S.: Mathematical theory of Lyapunov exponents. J. Phys. A Math. Theor. 46(25), 254001 (2013)

95. Pikovsky, A., Politi, A.: Lyapunov Exponents: A Tool to Explore Complex Dynamics. Cambridge University Press, Cambridge (2016)

96. Kuznetsov, N., Leonov, G.: On stability by the first approximation for discrete systems. In: 2005 International Conference on Physics and Control, PhysCon 2005. Volume Proceedings Volume 2005. IEEE, pp. 596-599 (2005)

97. Leonov, G., Kuznetsov, N.: Time-varying linearization and the Perron effects. Int. J. Bifurc. Chaos 17(4), 1079-1107 (2007)

98. Benedicks, M., Young, L.S.: Sinai-Bowen-Ruelle measures for certain Henon maps. Invent. Math. 112(1), 541$576(1993)$

99. Tasaki, S., Gilbert, T., Dorfman, J.: An analytical construction of the SRB measures for Baker-type maps. Chaos Interdiscip. J. Nonlinear Sci. 8(2), 424-443 (1998)

100. Schmeling, J.: A dimension formula for endomorphismsthe Belykh family. Ergod. Theory Dyn. Syst. 18, 12831309 (1998)

101. Kuznetsov, N., Leonov, G., Mokaev, T.: Finite-time and exact Lyapunov dimension of the Henon map (2017). arxiv: 1712.01270

102. Kehlet, B., Logg, A.: Quantifying the computability of the Lorenz system using a posteriori analysis. In: Proceedings of the VI International Conference on Adaptive Modeling and Simulation (ADMOS 2013) (2013)

103. Kehlet, B., Logg, A.: A posteriori error analysis of roundoff errors in the numerical solution of ordinary differential equations. Numer. Algorithms 76(1), 191-210 (2017)

104. Liao, S., Wang, P.: On the mathematically reliable longterm simulation of chaotic solutions of Lorenz equation in the interval $[0,10000]$. Sci. China Phys. Mech. Astron. 57(2), 330-335 (2014)
105. Eden, A.: An abstract theory of L-exponents with applications to dimension analysis. Ph.D. thesis. Indiana University (1989)

106. Ramasubramanian, K., Sriram, M.: A comparative study of computation of Lyapunov spectra with different algorithms. Phys. D Nonlinear Phenom. 139(1-2), 72-86 (2000)

107. Rutishauser, H., Schwarz, H.: The LR transformation method for symmetric matrices. Numer. Math. 5(1), 273$289(1963)$

108. Stewart, D.: A new algorithm for the SVD of a long product of matrices and the stability of products. Electron. Trans. Numer. Anal. 5, 29-47 (1997)

109. Benettin, G., Galgani, L., Giorgilli, A., Strelcyn, J.M.: Lyapunov characteristic exponents for smooth dynamical systems and for hamiltonian systems. A method for computing all of them. Part 2: Numerical application. Meccanica 15(1), 21-30 (1980)

110. Wolf, A., Swift, J.B., Swinney, H.L., Vastano, J.A.: Determining Lyapunov exponents from a time series. Phys. D Nonlinear Phenom. 16(D), 285-317 (1985)

111. Bylov, B.E., Vinograd, R.E., Grobman, D.M., Nemytskii, V.V.: Theory of Characteristic Exponents and Its Applications to Problems of Stability. Nauka, Moscow (1966). (in Russian)

112. Smith, R.: Some application of Hausdorff dimension inequalities for ordinary differential equation. Proc. R. Soc. Edinb. 104A, 235-259 (1986)

113. Doering, C., Gibbon, J., Holm, D., Nicolaenko, B.: Exact Lyapunov dimension of the universal attractor for the complex Ginzburg-Landau equation. Phys. Rev. Lett. 59, 2911-2914 (1987)

114. Kuznetsov, N., Leonov, G.: A short survey on Lyapunov dimension for finite dimensional dynamical systems in Euclidean space (2016). arxiv: 1510.03835

115. Leonov, G.: On estimations of Hausdorff dimension of attractors. Vestnik St. Petersb. Univ. Math. 24(3): 3841 (1991) [Transl. from Russian: Vestnik Leningradskogo Universiteta. Mathematika, 24(3): 41-44 (1991)]

116. Boichenko, V., Leonov, G.: Lyapunov's direct method in estimates of topological entropy. J. Math. Sci. 91(6), 33703379 (1998)

117. Pogromsky, A., Matveev, A.: Estimation of topological entropy via the direct Lyapunov method. Nonlinearity 24(7), 1937-1959 (2011)

118. Kuznetsov, N., Mokaev, T., Vasilyev, P.: Numerical justification of Leonov conjecture on Lyapunov dimension of Rossler attractor. Commun. Nonlinear Sci. Numer. Simul. 19, 1027-1034 (2014)

119. Hurewicz, W., Wallman, H.: Dimension Theory. Princeton University Press, Princeton (1941)

120. Siu, S. www.mathworks.com/matlabcentral/fileexchange/ 233-let (1998) 